\title{
Calmness of Linear Constraint Systems under Structured Perturbations with an Application to the Path-Following Scheme
}

\author{
C. Argáez ${ }^{1}$ - M.J. Cánovas ${ }^{2} \cdot$ J. Parra ${ }^{2}$ (D)
}

Received: 29 July 2020 / Accepted: 21 June 2021 / Published online: 14 July 2021

(C) The Author(s) 2021

\begin{abstract}
We are concerned with finite linear constraint systems in a parametric framework where the right-hand side is an affine function of the perturbation parameter. Such structured perturbations provide a unified framework for different parametric models in the literature, as block, directional and/or partial perturbations of both inequalities and equalities. We extend some recent results about calmness of the feasible set mapping and provide an application to the convergence of a certain path-following algorithmic scheme. We underline the fact that our formula for the calmness modulus depends only on the nominal data, which makes it computable in practice.
\end{abstract}

Keywords Calmness · Linear systems of equalities and inequalities · Primal-dual path-following algorithm $\cdot$ Linear programming $\cdot$ Feasible set mapping

Mathematics Subject Classification (2010) 90C31 · 49J53 • 90C05 • 90C51

\section{Introduction and Overview}

The present paper deals with parameterized linear inequality systems in $\mathbb{R}^{n}$ of the form

$$
\left\{a_{t}^{\prime} x \leq q_{t}+p_{t}^{\prime} b, t \in T:=\{1, \ldots, m\}\right\},
$$

Dedicated to R.T. Rockafellar on his 85 th birthday

J. Parra

parra@umh.es

C. Argáez

carlos@hi.is

M.J. Cánovas

canovas@umh.es

1 Faculty of Physical Sciences, University of Iceland, Reykjavik, Iceland

2 Center of Operations Research, Miguel Hernández University of Elche, 03202

Elche (Alicante), Spain 
where $x \in \mathbb{R}^{n}$ is the vector of decision variables regarded as a column vector (i.e. $\mathbb{R}^{n} \equiv$ $\mathbb{R}^{n \times 1}$ ), the prime stands for transposition, $a_{t} \in \mathbb{R}^{n}, p_{t} \in \mathbb{R}^{k}$ and $q_{t} \in \mathbb{R}$ are given (fixed) for each index $t \in T$, and $b \in \mathbb{R}^{k}$ is the parameter to be perturbed around a nominal (or reference) element $\bar{b}$. Alternatively we could use the following matrix notation

$$
A x \leq q+P b,
$$

where $A \in \mathbb{R}^{m \times n}$ and $P \in \mathbb{R}^{m \times k}$ stand for the matrices whose $t$-th rows are, respectively $a_{t}^{\prime}$ and $p_{t}^{\prime}$, and $q=\left(q_{t}\right)_{t \in T} \in \mathbb{R}^{T} \equiv \mathbb{R}^{m}$. The natural numbers $n, m$, and $k$ remain fixed throughout the paper. This framework of structured perturbations includes as particular cases some previously analyzed contexts as the usual right-hand side (RHS for short) perturbation setting, where $q=0_{m}$ (the zero vector of $\mathbb{R}^{m}$ ) and $P=I_{m}$ (the identity matrix of order $m$ ); see, e.g., $[11,12]$. It also allows for block perturbations as in [9] (see Example 1 below) or unperturbed inequalities as in [6] (see Example 3 including also equalities); moreover, it includes more sophisticated cases as the one of Example 2, dealing with multiobjective linear programs, traced out from [10].

The presence of $q$ in the model allows the nominal right-hand side $q+P \bar{b}$ to be any vector of $\mathbb{R}^{m}$, not necessarily in the range of $P$. Of course we could consider $\bar{b}=0_{k}$, but we prefer to keep an arbitrarily fixed $\bar{b}$ in accordance with the notation of the aforementioned antecedents. The model (1) makes also sense when $T$ is an arbitrary (possible infinite) index set; in this case we are dealing with linear semi-infinite systems, extensively studied in [15].

The main goal of the paper concerns the calmness modulus (see Section 2 for definitions) of the feasible set mapping $\mathcal{F}: \mathbb{R}^{k} \rightrightarrows \mathbb{R}^{n}$, given by

$$
\mathcal{F}(b):=\left\{x \in \mathbb{R}^{n}: A x \leq q+P b\right\}, \text { for } b \in \mathbb{R}^{k} .
$$

The continuity properties of $\mathcal{F}$ in the context of RHS perturbations (with $q=0_{m}$ and $P=I_{m}$ ) have been studied, for instance, in [15, Chapter 6], whereas the computation of the calmness modulus of $\mathcal{F}$ in this particular setting has been carried out in [11, Section 4].

At this moment, let us exhibit some of the aforementioned particular cases of our model (1)-(2). Example 3 will be the basis for our application to the path-following scheme in Section 4. Accordingly, this example will be analyzed more in detail in relation to calmness in Section 3.2.

Example 1 (Block perturbations) We consider a partition $\left\{T_{1}, \ldots, T_{k}\right\}$ of $T$ and all constraints of the same block $T_{j}$, for $j=1, \ldots, k$, are perturbed with the same $b_{j}$. This fits model (2) if the $j$-th column of $P$ consists of 1 in those rows $t \in T_{j}$ and 0 otherwise. The extreme cases are $P=I_{m}$, when $T_{j}=\{j\}$, commented above, and the case of 'constant perturbations', corresponding to $T_{1}=T$. See further comments and references in [9, p. 311].

Example 2 (Epigraphical feasible sets) Paper [10] is concerned with the Lipschitzian behavior of the so-called Pareto front mapping associated with the multiobjective linear programming (MLP) problem given by

$$
\begin{aligned}
M L P(b): & \operatorname{minimize}\left(c_{1}^{\prime} x, \ldots, c_{s}^{\prime} x\right) \\
& \text { subject to } A x \leq b,
\end{aligned}
$$

(adapted to our current notation) where $c_{1}, \ldots, c_{s} \in \mathbb{R}^{n}$ are fixed. Denoting by $\mathcal{F}(b)$ the feasible set of system $A x \leq b,[10]$ considers the so-called epigraphical feasible set mapping $\mathcal{E}_{\mathcal{F}}: \mathbb{R}^{m} \rightrightarrows \mathbb{R}^{n}$ given by

$$
\mathcal{E}_{\mathcal{F}}(b):=\mathcal{F}(b)+\left\{c_{1}, \ldots, c_{s}\right\}^{\circ},
$$


where $\Omega^{\circ}:=\left\{y \in \mathbb{R}^{n}: y^{\prime} x \geq 0\right.$ for all $\left.x \in \Omega\right\}$ stands for the (positive) polar cone to the set $\Omega \subset \mathbb{R}^{n}$. Specifically, [10, Section 4] establishes a finite iterative procedure to express $\mathcal{E}_{\mathcal{F}}(b)$ as the feasible set of a linear inequality system of the form (2) with $q=0_{m}$. This is illustrated in [10, Example 3].

Example 3 (Including equations and unperturbed constraints) Let us consider the model

$$
\left\{A_{1} x \leq b_{1}, A_{2} x=b_{2}, A_{3} x \leq b_{3}^{0}\right\},
$$

with $A_{i} \in \mathbb{R}^{m_{i} \times n}$ for $i=1,2,3$ and $b_{3}^{0} \in \mathbb{R}^{m_{3}}$ being fixed, and parameters $b_{i} \in \mathbb{R}^{m_{i}}$, with $i=1,2$. This case fits model (2) with $k=m_{1}+m_{2}, m=m_{1}+2 m_{2}+m_{3}$, and

$$
\left.A=\left(\begin{array}{c}
A_{1} \\
A_{2} \\
-A_{2} \\
A_{3}
\end{array}\right), \quad P=\left(\begin{array}{cc}
I_{m_{1}} & 0_{m_{1} \times m_{2}} \\
0_{m_{2} \times m_{1}} & I_{m_{2}} \\
0_{m_{2} \times m_{1}} & -I_{m_{2}} \\
0_{m_{3} \times m_{1}} & 0_{m_{3} \times m_{2}}
\end{array}\right), \quad \begin{array}{c}
\mathrm{b}= \\
\mathrm{q}=\left(\begin{array}{c}
b_{1} \\
b_{2}
\end{array}\right), \\
0_{m_{1}+2 m_{2}} \\
b_{3}^{0}
\end{array}\right),
$$

where $0_{i \times j}$ stands for the zero matrix of size $i \times j$.

The aim of this work is twofold. Firstly, Section 3 is focussed on computing the calmness modulus of mapping $\mathcal{F}$ introduced in (3). This section is divided into two parts dealing, respectively, with a generic approach via the analysis of local error bounds and outer limit of subdifferentials (appealing to [24, Theorem 1]), and with a more specific one tackling the calmness modulus of $\mathcal{F}$ from its definition. For some particular choices of matrix $P$ we succeed to provide point-based expressions (depending exclusively on the nominal data) for the aimed modulus. This is the case of model (4), which plays a remarkable role in this work because of its application to Section 4, and we point out that the results of this paper extend the calmness analysis developed in $[6,11]$, as far as it includes not only inequalities, but also equalities. It is worth mentioning that, in general, the stability study of linear systems in presence of equalities cannot be directly derived from the study of linear inequality systems by just splitting each equality into two inequalities. This fact has been frequently pointed out in the literature in different frameworks; see e.g. the classical work of [26, p. 37] or the more recent one [5]. As a simple example, consider a consistent linear equality in $\mathbb{R}^{n}$ given by ' $a$ ' $x=b$ ', with $a \neq 0_{n}$, split as ' $a$ ' $x \leq b,-a^{\prime} x \leq-b$ ', and observe that the perturbed equality ' $a$ ' $x=b-\varepsilon$ ' is consistent for all $\varepsilon \in \mathbb{R}$, while the perturbed system ' $a$ ' $x \leq b-\varepsilon,-a^{\prime} x \leq-b-\varepsilon$ ' is no longer consistent whenever $\varepsilon>0$. In relation to this concern, the present work shows that this splitting strategy does work when confined to the analysis of the calmness property for model (4).

Secondly, Section 4 introduces a certain feasible set mapping $\mathcal{N}$ of a parametric constraint system of the form (4) whose image at the nominal parameter coincides with the set of all primal-dual solutions to a linear programming (LP) problem in $\mathbb{R}^{n}$. Specifically, this section shows how the calmness modulus of $\mathcal{N}$ enables to quantify the speed of convergence of a path-following scheme devoted to solve such a problem.

The translation of calmness and other Lipschitzian constants into measures of convergence of algorithms has been tackled by different authors; see, e.g., [2, 14, 21-23, 35]. Our application is inspired by [28] (see also [21, p. 31]). In relation to this point, the original contribution of this work consists in the fact that the provided constants are computable as far as they are expressed in terms of the given data.

In summary, the paper is structured as follows: Section 2 provides the necessary notation, definitions and preliminary results. Section 3 computes or estimates the calmness modulus 
of $\mathcal{F}$ in context (1)-(2), paying attention to special cases of $P$ for which a point based formula of the modulus is obtained, which is the case of (4). The latter is applied in Section 4 to the aforementioned path-following scheme.

\section{Preliminaries and First Results}

In order to measure the size of point/parameter perturbations, the space of variables, $\mathbb{R}^{n}$, is equipped with an arbitrary norm, $\|\cdot\|$, whose corresponding dual norm is given by $\|u\|_{*}=\max _{\|x\| \leq 1}\left|u^{\prime} x\right|$. Perturbations of parameter $b \in \mathbb{R}^{k}$ are measured by means of the supremum (Chebyshev) norm, $\|b\|_{\infty}:=\max _{j=1, \ldots, k}\left|b_{j}\right|$, whose dual norm is denoted by $\|b\|_{1}\left(:=\sum_{i=1}^{k}\left|b_{j}\right|\right)$.

Given $X \subset \mathbb{R}^{p}, p \in \mathbb{N}$, we denote by $\operatorname{conv} X$, cone $X$, and span $X$ the convex hull, the conical convex hull, and the linear subspace generated by $X$, respectively. It is assumed that cone $X$ and $\operatorname{span} X$ always contain the zero-vector, $0_{p}$, in particular cone $(\emptyset)=\operatorname{span}(\emptyset)=$ $\left\{0_{p}\right\}$, whereas $\operatorname{conv}(\emptyset)=\emptyset$. If $X$ is a subset of any topological space, int $X, \operatorname{cl} X$ and $\operatorname{bd} X$ stand, respectively, for the interior, the closure and the boundary of $X$. For a nonempty set $S, \mathbb{R}^{S}$ and $\mathbb{R}_{+}^{S}$ denote the sets of all functions from $S$ to $\mathbb{R}$ and $\mathbb{R}_{+}:=[0,+\infty[$, respectively.

A set-valued mapping $\mathcal{M}: Y \rightrightarrows X$ between metric spaces (with $d$ denoting both distances) is said to be calm at $(\bar{y}, \bar{x}) \in \operatorname{gph} \mathcal{M}$ (the graph of $\mathcal{M}$ ) if there exist $\kappa \geq 0$ and neighborhoods $W$ of $\bar{x}$ and $V$ of $\bar{y}$ such that

$$
d(x, \mathcal{M}(\bar{y})) \leq \kappa d(y, \bar{y})
$$

whenever $x \in \mathcal{M}(y) \cap W$ and $y \in V$, where, as usual, $d(x, X):=\inf \{d(x, z): z \in X\}$ for $X \subset \mathbb{R}^{n}$, and $d(x, \emptyset):=+\infty$. We shall refer to any constant $\kappa \geq 0$ verifying (5), for some associated neighborhoods, as a calmness constant.

It is well-known that the calmness of $\mathcal{M}$ is equivalent to the metric subregularity of the inverse multifunction $\mathcal{M}^{-1}: X \rightrightarrows Y$, given by $\mathcal{M}^{-1}(x):=\{y \in Y \mid x \in \mathcal{M}(y)\}$ (see, for instance, [13, Section 3H]), which means the existence of $\kappa \geq 0$ and a (possibly smaller) neighborhood $W$ of $\bar{x}$ such that

$$
d(x, \mathcal{M}(\bar{y})) \leq \kappa d\left(\bar{y}, \mathcal{M}^{-1}(x)\right)
$$

for all $x \in W$. The infimum of all possible constants $\kappa$ satisfying (5) (for some associated $W$ and $V$ ) equals the infimum of those satisfying (6) and is called the calmness modulus of $\mathcal{M}$ at $(\bar{y}, \bar{x})$, denoted as $\operatorname{clm} \mathcal{M}(\bar{y}, \bar{x})$, defined as $+\infty$ if $\mathcal{M}$ is not calm at $(\bar{y}, \bar{x})$. More in detail, later on we will use the following expression, which comes straightforwardly from the definitions (under the convention $0 / 0:=0$ ):

$$
\operatorname{clm} \mathcal{M}(\bar{y}, \bar{x})=\limsup _{\substack{(y, x) \rightarrow(\bar{y}, \bar{x}) \\ x \in \mathcal{M}(y)}} \frac{d(x, \mathcal{M}(\bar{y}))}{d(y, \bar{y})}=\limsup _{x \rightarrow \bar{x}} \frac{d(x, \mathcal{M}(\bar{y}))}{d\left(\bar{y}, \mathcal{M}^{-1}(x)\right)}
$$

The calmness property plays a central role in many issues of mathematical programming (optimality conditions, error bounds, stability of solutions...); the reader is addressed to the monographs $[13,19,21,29,34]$ for a comprehensive study of this and other variational properties. For more specific comments and references we refer to [6, p. 466]. 


\subsection{Calmness, Error Bounds and Outer Limit of Subdifferentials}

Let us recall that an extended real-valued function $f: \mathbb{R}^{n} \rightarrow \mathbb{R} \cup\{+\infty\}$ is said to admit a local error bound at $\bar{x} \in \mathbb{R}^{n}$ if

$$
\tau d(x,[f \leq 0]) \leq[f(x)]_{+}
$$

for a certain $\tau>0$ and for all $x$ in a certain neighborhood $W$ of $\bar{x}$, where $[f \leq 0]:=$ $\left\{x \in \mathbb{R}^{n}: f(x) \leq 0\right\}$ and $[\alpha]_{+}:=\max \{\alpha, 0\}, \alpha \in \mathbb{R}$. The supremum of those $\tau>0$ satisfying (8) (for some related neighborhood $W$ ) is called the error bound modulus of $f$ at $\bar{x} \in \mathbb{R}^{n}$, denoted by $\operatorname{Er} f(\bar{x})$ (defined as 0 if $f$ does not have a local error bound at $\bar{x}$ ). The following result can be traced out from [24, Theorem 1].

Theorem 1 Let $f: \mathbb{R}^{n} \rightarrow \mathbb{R} \cup\{+\infty\}$ be a proper lower semicontinuous convex function and $\bar{x} \in \mathbb{R}^{n}$ be a point such that $f(\bar{x})=0$. Then $f$ admits a local error bound at $\bar{x}$ if and only if

$$
\liminf _{x \rightarrow \bar{x}, f(x)>0} d_{*}\left(0_{n}, \partial f(x)\right)>0 .
$$

Moreover, under this condition,

$$
\operatorname{Er} f(\bar{x})=\liminf _{x \rightarrow \bar{x}, f(x)>0} d_{*}\left(0_{n}, \partial f(x)\right),
$$

where $d_{*}$ stands for the distance in $\mathbb{R}^{n}$ associated with $\|\cdot\|_{*}$ and $\partial$ represents the usual subdifferential of convex analysis.

Taking into account that, for any $\bar{x} \in \mathbb{R}^{n}$ such that $f(\bar{x})=0$,

$$
\liminf _{x \rightarrow \bar{x}, f(x)>0} d_{*}\left(0_{n}, \partial f(x)\right)=d_{*}\left(0_{n}, \limsup _{x \rightarrow \bar{x}, f(x)>0} \partial f(x)\right),
$$

where the lim sup is understood in the Painlevé-Kuratowski sense (see [29, p. 3] and [34, Corollary 4.7(b)]), the outer limit of subdifferentials,

$$
\partial^{>} f(\bar{x}):=\lim \sup _{x \rightarrow \bar{x}}, f(x)>0 \partial f(x),
$$

constitutes a key tool in the computation of error bound moduli.

Let us recall the relationship between local error bounds and calmness moduli for specific multifunctions. Liking with the notation of [6, Section 3.2] let us consider the feasible set mapping $\mathcal{F}_{I, U}: \mathbb{R}^{I} \rightrightarrows \mathbb{R}^{n}$ given by

$$
\mathcal{F}_{I, U}(b):=\left\{x \in \mathbb{R}^{n}: a_{t}^{\prime} x \leq b_{t}, t \in I ; a_{t}^{\prime} x \leq b_{t}^{0}, t \in U\right\},
$$

where $I$ (after 'inequalities') and $U$ (after 'unperturbed') are disjoint finite sets, and $b=$ $\left(b_{t}\right)_{t \in I} \in \mathbb{R}^{I}$. Fix $\bar{b} \in \mathbb{R}^{I}$ and consider the associated supremum function $s_{\bar{b}}: \mathbb{R}^{n} \rightarrow$ $\mathbb{R} \cup\{+\infty\}$ given by

$$
s_{\bar{b}}(x):=\max _{t \in I}\left(a_{t}^{\prime} x-\bar{b}_{t}\right)+\mathrm{I}_{C}(x),
$$

where $C=\left\{x \in \mathbb{R}^{n} \mid a_{t}^{\prime} x \leq b_{t}^{0}, t \in U\right\}$ and $\mathrm{I}_{C}$ is the indicator function:

$$
\mathrm{I}_{C}(x):=\left\{\begin{array}{c}
0, \text { if } x \in C, \\
+\infty, \text { if } x \in \mathbb{R}^{n} \backslash C .
\end{array}\right.
$$

Remark 1 Take $\bar{x} \in \mathcal{F}_{I, U}(\bar{b})$ and observe that $\mathcal{F}_{I, U}(\bar{b})=\left[s_{\bar{b}} \leq 0\right]$ and $d\left(\bar{b}, \mathcal{F}_{I, U}^{-1}(x)\right)=$ $\left[s_{\bar{b}}(x)\right]_{+}, x \in \mathbb{R}^{n}$. Hence the calmness of $\mathcal{F}_{I, U}$ at $(\bar{b}, \bar{x})$, turns out to be equivalent (recall 
(6)) to the existence of a local error bound for $s_{\bar{b}}$ at $\bar{x}$. Moreover,

$$
\operatorname{clm}_{\mathcal{F}_{I, U}}(\bar{b}, \bar{x})=\left(\operatorname{Er} s_{\bar{b}}(\bar{x})\right)^{-1}=d_{*}\left(0_{n}, \partial^{>} s_{\bar{b}}(\bar{x})\right)^{-1} .
$$

Next we recall a point based formula for $\partial^{>} s_{\bar{b}}(x)$, and so for $\operatorname{clm} \mathcal{F}_{I, U}(\bar{b}, \bar{x})$ in the nontrivial case $s_{\bar{b}}(\bar{x})=0\left(s_{\bar{b}}(\bar{x})<0\right.$ implies $\operatorname{clm} \mathcal{F}_{I, U}(\bar{b}, \bar{x})=0$ and $\left.\operatorname{Er}_{\bar{b}}(\bar{x})=+\infty\right)$. Consider the subsets of active indices

$$
I_{\bar{b}}(\bar{x}):=\left\{t \in I \mid a_{t}^{\prime} \bar{x}=b_{t}\right\} \text { and } U(\bar{x}):=\left\{t \in U \mid a_{t}^{\prime} \bar{x}=b_{t}^{0}\right\},
$$

and define the family $\mathcal{I}(\bar{x})$ formed by all pairs of subsets of indices of the form $\left(I_{1}, U_{1}\right)$ with $I_{1} \subset I_{\bar{b}}(\bar{x})$ and $U_{1} \subset U(\bar{x})$ such that the following system has a solution in $d \in \mathbb{R}^{n}$ :

$$
\left\{\begin{array}{l}
a_{t}^{\prime} d=1, t \in I_{1}, \\
a_{t}^{\prime} d<1, t \in I_{\bar{b}}(\bar{x}) \backslash I_{1}, \\
a_{t}^{\prime} d=0, t \in U_{1}, \\
a_{t}^{\prime} d<0, t \in U(\bar{x}) \backslash U_{1} .
\end{array}\right.
$$

Family $\mathcal{I}(\bar{x})$ comes from extending [11, Section 4] and adapting (and, roughly speaking, normalizing with 1) its counterpart in [6, Section 3.2] (taking [33, Theorem 23.4] into account for computing the directional derivatives therein).

Theorem 2 [6, Theorem 3.4] Consider $\bar{x} \in \mathcal{F}_{I, U}(\bar{b})$ with $s_{\bar{b}}(\bar{x})=0$. Then,

$$
\partial^{>} s_{\bar{b}}(\bar{x})=\bigcup_{\left(I_{1}, U_{1}\right) \in \mathcal{I}(\bar{x})}\left(\operatorname{conv}\left\{a_{t}, t \in I_{1}\right\}+\operatorname{cone}\left\{a_{t}, t \in U_{1}\right\}\right) .
$$

Consequently,

$$
\operatorname{clm} \mathcal{F}_{I, U}(\bar{b}, \bar{x})=\left(\min _{\left(I_{1}, U_{1}\right) \in \mathcal{I}(\bar{x})} d_{*}\left(0_{n}, \operatorname{conv}\left\{a_{t}, t \in I_{1}\right\}+\operatorname{cone}\left\{a_{t}, t \in U_{1}\right\}\right)\right)^{-1} .
$$

Remark 2 Observe that in the statement of the previous theorem we could confine ourselves to those $\left(I_{1}, U_{1}\right) \in \mathcal{I}(\bar{x})$ which are maximal with respect to the (coordinatewise) inclusion order. We do not exclude the possibility $I_{1}=\emptyset$ or $U_{1}=\emptyset$, recalling $\operatorname{conv}(\emptyset)=\emptyset$ and cone $(\emptyset)=\left\{0_{n}\right\}$, under the convention $\emptyset+X=\emptyset$ for $X \subset \mathbb{R}^{n}$ and $d_{*}\left(0_{n}, \emptyset\right)=+\infty$.

The next remark appeals to the concept of end of a nonempty convex set $C \subset \mathbb{R}^{n}$ introduced in [17] (see also [25]), defined as

$$
\text { end } C:=\{u \in \operatorname{cl} C: \nexists \mu>1 \text { such that } \mu u \in \operatorname{cl} C\} \text {. }
$$

Remark 3 The case when $U=\emptyset$ was analyzed in [11, Section 4], where a family $\mathcal{D}(\bar{x})$ of subsets $I_{1} \subset I_{\bar{b}}(\bar{x})$ was introduced through the existence of a solution $d \in \mathbb{R}^{n}$ of the system formed by the two first blocks of (13). Hence, in this particular case, (14) reads as

$$
\partial^{>} s_{\bar{b}}(\bar{x})=\bigcup_{I_{1} \in \mathcal{D}(\bar{x})} \operatorname{conv}\left\{a_{t}, t \in I_{1}\right\}=\operatorname{end} \operatorname{conv}\left\{a_{t}, t \in I_{\bar{b}}(\bar{x})\right\} .
$$

The first equality above was already given in [7, Theorem 3.1].

The following example is intended to illustrate Theorem 2 . 
Example 4 Consider the systems in $\mathbb{R}$ and $\mathbb{R}^{2}$ (with the Euclidean norm)

$$
\text { (i) } \left.\left\{\begin{aligned}
x \leq b, t=1 \in I \\
-x \leq 0, t=2 \in U
\end{aligned}\right\}, \quad \text { (ii }\right) \quad\left\{\begin{array}{ll}
x_{1}+x_{2} \leq b, t=1 \in I \\
x_{1} \leq 0, & t=2 \in U \\
x_{2} \leq 0, & t=3 \in U
\end{array}\right\} \text {. }
$$

Then, in $(i)$ for $(\bar{b}, \bar{x})=(0,0)$ we have $\mathcal{I}(0)=\{(\{1\}, \emptyset),(\emptyset,\{2\})\}$ and $\operatorname{clm} \mathcal{F}_{I, U}(\bar{b}, \bar{x})=1$.

In $(i i)$ for $(\bar{b}, \bar{x})=\left(0,0_{2}\right)$ we have $\mathcal{I}\left(0_{2}\right)=\left\{\left(\emptyset, U_{1}\right): U_{1} \subset\{1,2\}\right\}$ and $\operatorname{clm} \mathcal{F}_{I, U}(\bar{b}, \bar{x})=(+\infty)^{-1}=0$.

Remark 4 Although from a theoretical point of view it is simpler that all constraints with index $t \in U$ in (10) be inequalities (by splitting unperturbed equalities $a_{t}^{\prime} x=b_{t}^{0}$, if any, into $a_{t}^{\prime} x \leq b_{t}^{0}$ and $-a_{t}^{\prime} x \leq-b_{t}^{0}$ ), we can observe that if $t_{1}$ and $t_{2}$ are the new indices associated with these split inequalities, then from (13) it must be $\left\{t_{1}, t_{2}\right\} \subset U_{1}$ for all $\left(I_{1}, U_{1}\right) \in \mathcal{I}(\bar{x})$. Alternatively, we could leave unperturbed equalities as equalities by replacing the corresponding cone $\left\{ \pm a_{t}\right\}$ with span $\left\{a_{t}\right\}$ in Theorem 2. This remark will be useful in Example 6 at the end of Section 4.

\section{Calmness Modulus of Constraint Systems under Structured Perturbations}

This section is devoted to computation of the calmness modulus of the feasible set mapping $\mathcal{F}$ introduced in $(3)$ at $(\bar{b}, \bar{x}) \in \operatorname{gph} \mathcal{F}, \operatorname{clm} \mathcal{F}(\bar{b}, \bar{x})$. This is tackled through two different approaches gathered into two subsections. Firstly, we define an appropriate function whose error bound modulus (its inverse, in fact) provides the aimed $\operatorname{clm} \mathcal{F}(\bar{b}, \bar{x})$. Hence, we apply Theorem 1. By following this theoretical approach, Section 3.1 finishes with a particular case of matrix $P$ for which an implementable point based formula is obtained. Section 3.2 approaches the computation of $\operatorname{clm} \mathcal{F}(\bar{b}, \bar{x})$ through its definition and is focussed on the relationship between this modulus and the one of the feasible mapping in the framework of RHS perturbations introduced in (10), which was previously studied in [6] (see Theorem 2). Special attention is paid to the models of Examples 1 and 3, where the exact formula for $\operatorname{clm} \mathcal{F}(\bar{b}, \bar{x})$ is derived by using Theorem 2 .

\subsection{A Generic Approach via Outer Limits of Subdifferentials}

Along this subsection we assume $P \neq 0_{m \times k}$; otherwise $\mathcal{F}$ is constant and, trivially, $\operatorname{clm} \mathcal{F}(\bar{b}, \bar{x})=0$. Define $m_{\bar{b}}: \mathbb{R}^{n} \rightarrow \mathbb{R}_{+} \cup\{+\infty\}$ by:

$$
m_{\bar{b}}(x):=\sup _{\lambda \in \Lambda} \frac{(-P \bar{b}-q+A x)^{\prime} \lambda}{\left\|P^{\prime} \lambda\right\|_{1}}, x \in \mathbb{R}^{n},
$$

where $\Lambda:=\left\{\lambda=\left(\lambda_{i}\right)_{i=1}^{m} \in \mathbb{R}_{+}^{m} \mid \sum_{i=1}^{m} \lambda_{i}=1\right\}$ is the standard $(m-1)$-simplex in $\mathbb{R}^{m}$; the possibilities $-\infty$ and $+\infty$ are not excluded for some ratios appearing in (15) (they correspond to $\frac{\alpha}{0}$ with $\alpha<0$ and $\alpha>0$, respectively; recall $\left.\frac{0}{0}=0\right)$. It is clear that

$$
m_{\bar{b}}(x)>-\infty \text { for all } x \in \mathbb{R}^{n},
$$

since $P \neq 0_{m \times k}$ entails the existence of $\lambda_{0} \in \Lambda$, such that $\left\|P^{\prime} \lambda_{0}\right\|_{1}>0$ and then $m_{\bar{b}}(x) \geq$ $\left\|P^{\prime} \lambda_{0}\right\|_{1}^{-1}(A x-P \bar{b}-q)^{\prime} \lambda_{0}>-\infty$. 
The following lemma gathers some properties of $m_{\bar{b}}$. Recall that the domain of a multifunction $\mathcal{M}: Y \rightrightarrows X$ is defined as $\operatorname{dom} \mathcal{M}:=\{y \in Y: \mathcal{M}(y) \neq \emptyset\}$.

Lemma 1 If $x \in \operatorname{dom} \mathcal{F}^{-1}$, then:

(i) $\left[m_{\bar{b}}(x)\right]_{+}=d\left(\bar{b}, \mathcal{F}^{-1}(x)\right)$;

(ii) $m_{\bar{b}}(x)=\max _{\lambda \in \Lambda} \frac{(-P \bar{b}-q+A x)^{\prime} \lambda}{\left\|P^{\prime} \lambda\right\|_{1}}$, i.e., the supremum in (15) is attained.

Proof (i) Fix $x \in \operatorname{dom} \mathcal{F}^{-1}$. Since $\mathcal{F}^{-1}(x)=\left\{b \in \mathbb{R}^{k}:-P b \leq q-A x\right\} \neq \emptyset$, applying [12, Lemma 1], we have

$$
d\left(\bar{b}, \mathcal{F}^{-1}(x)\right)=\max _{\lambda \in \mathbb{R}_{+}^{m}} \frac{\left[(-P \bar{b}-q+A x)^{\prime} \lambda\right]_{+}}{\left\|P^{\prime} \lambda\right\|_{1}} .
$$

Then, we only have to check that ' $\lambda \in \mathbb{R}_{+}^{m}$ ' in (16) may be replaced with ' $\lambda \in \Lambda$ '. In fact, if $d\left(\bar{b}, \mathcal{F}^{-1}(x)\right)=0$, one has $-P \bar{b}-q+A x \leq 0_{m}$ and it is clear that the maximum in (16) is attained at any $\lambda \in \Lambda$. On the other hand, if $d\left(\bar{b}, \mathcal{F}^{-1}(x)\right)>0$ and the maximum in (16) is attained at $\bar{\lambda} \in \mathbb{R}_{+}^{m}$, then $\bar{\lambda} \neq 0_{m}$ and the same maximum is also attained at $\left(\sum_{i=1}^{m} \bar{\lambda}_{i}\right)^{-1} \bar{\lambda} \in \Lambda$.

(ii) If $m_{\bar{b}}(x)>0$ the results follows as $(i)$. If $m_{\bar{b}}(x) \leq 0$, write:

$$
m_{\bar{b}}(x)=\lim _{r} \frac{(-P \bar{b}-q+A x)^{\prime} \lambda^{r}}{\left\|P^{\prime} \lambda^{r}\right\|_{1}},
$$

for some sequence $\left\{\lambda^{r}\right\} \subset \Lambda$ which is assumed to converge to certain $\widehat{\lambda}$ (by the compactness of $\Lambda$ ). Then, one has

$$
\lim _{r}(-P \bar{b}-q+A x)^{\prime} \lambda^{r}=(-P \bar{b}-q+A x)^{\prime} \widehat{\lambda} \leq 0,
$$

and we distinguish two cases: if $(-P \bar{b}-q+A x)^{\prime} \widehat{\lambda}=0$, it is clear that $m_{\bar{b}}(x)=0$ and the supremum (15) is attained at $\widehat{\lambda}$. If $(-P \bar{b}-q+A x)^{\prime} \widehat{\lambda}<0$, then $P^{\prime} \widehat{\lambda} \neq 0_{k}$ (otherwise $m_{\bar{b}}(x)=-\infty$, which is impossible) and

$$
m_{\bar{b}}(x)=\frac{(-P \bar{b}-q+A x)^{\prime} \widehat{\lambda}}{\left\|P^{\prime} \widehat{\lambda}\right\|_{1}} .
$$

The next proposition provides the function whose error bound modulus does allow us to compute $\operatorname{clm} \mathcal{F}(\bar{b}, \bar{x})$.

Proposition 1 Let $f_{\bar{b}}: \mathbb{R}^{n} \rightarrow \mathbb{R}_{+} \cup\{+\infty\}$ be given by

$$
f_{\bar{b}}:=m_{\bar{b}}+\mathrm{I}_{\mathrm{dom} \mathcal{F}^{-1}} \text {. }
$$

We have:

(i) $f_{\bar{b}}$ is a proper lower semicontinuous convex function;

(ii) $\left[f_{\bar{b}}(x)\right]_{+}=d\left(\bar{b}, \mathcal{F}^{-1}(x)\right)$, for all $x \in \mathbb{R}^{n}$,

(iii) $\operatorname{clm} \mathcal{F}(\bar{b}, \bar{x})=\left(\operatorname{Er} f_{\bar{b}}(\bar{x})\right)^{-1}=d_{*}\left(0_{n}, \partial^{>} f_{\bar{b}}(\bar{x})\right)^{-1}$. 
Proof $(i)$ It is clear that $f_{\bar{b}}$ is a proper function since $m_{\bar{b}}(x)>-\infty$ for all $x \in \mathbb{R}^{n}$. We have

$$
f_{\bar{b}}(x)=m_{\bar{b}}(x)=\max _{\lambda \in \Lambda} \frac{(-P \bar{b}-q+A x)^{\prime} \lambda}{\left\|P^{\prime} \lambda\right\|_{1}} \text {, provided that } x \in \operatorname{dom} \mathcal{F}^{-1} \text {. }
$$

Hence $f_{\bar{b}}$ is finite valued and convex on the closed and convex set dom $\mathcal{F}^{-1}$. In fact, since gph $\mathcal{F}^{-1}=\left\{(x, b) \in \mathbb{R}^{m} \times \mathbb{R}^{k} \mid A x-P b \leq q\right\}$ is polyhedron, dom $\mathcal{F}^{-1}$ also is [33, Theorem 19.3]. Therefore, $f_{\bar{b}}$ is a lower semicontinuous convex function.

(i) comes from the definition of $f_{\bar{b}}$ taking the previous lemma into account.

(ii) By Theorem 1 and (9), it remains to check that

$$
\left[f_{\bar{b}} \leq 0\right]=\mathcal{F}(\bar{b}),
$$

which comes from the fact that, for any $x \in \mathbb{R}^{n}, f_{\bar{b}}(x) \leq 0$ if and only if $(-P \bar{b}-q+A x)^{\prime} \lambda \leq 0$ for all $\lambda \in \Lambda$ which is equivalent to $-P \bar{b}-q+A x \leq 0$; i.e., $x \in \mathcal{F}(\bar{b})$.

Remark 5 According to the previous proposition, to solve the problem of deriving an explicit formula for $\partial^{>} f_{\bar{b}}(\bar{x})$ for an arbitrary matrix $P \neq 0_{m \times k}$ constitutes a decisive step in the computation of $\operatorname{clm} \mathcal{F}(\bar{b}, \bar{x})$. The rest of this subsection deals with a particular $P$ for which $f_{\bar{b}}$ is polyhedral, and hence $\partial^{>} f_{\bar{b}}(\bar{x})$ is obtained as in Remark 3 . A key step here consists in replacing $\Lambda$ by a finite subset in the definition of $m_{\bar{b}}$. Outside this case, the computation of $\partial^{>} f_{\bar{b}}(\bar{x})$ through a point based formula remains as an open problem.

Proposition 2 Assume that $P^{\prime} \lambda \neq 0_{k}$ for all $\lambda \in \Lambda$; in other words $0_{k} \notin \operatorname{conv}\left\{p_{t}, t \in T\right\}$. Then, there exists a finite subset $\widetilde{\Lambda} \subset \Lambda$ such that

$$
f_{\bar{b}}(x)=\max _{\lambda \in \widetilde{\Lambda}} \frac{(-P \bar{b}-q+A x)^{\prime} \lambda}{\left\|P^{\prime} \lambda\right\|_{1}}, \text { for all } x \in \mathbb{R}^{n} .
$$

If $f_{\bar{b}}(\bar{x})=0$, then

$$
\partial^{>} f_{\bar{b}}(\bar{x})=\text { end conv }\left\{\frac{A^{\prime} \lambda}{\left\|P^{\prime} \lambda\right\|_{1}}: \lambda \in \tilde{\Lambda}, \quad(-P \bar{b}-q+A \bar{x})^{\prime} \lambda=0\right\} .
$$

Proof First, observe that under the current assumption, $\operatorname{dom} \mathcal{F}^{-1}=\mathbb{R}^{n}$ [15, Theorem 4.4]. Hence $f_{\bar{b}}(x)=m_{\bar{b}}(x)$, for all $x \in \mathbb{R}^{n}$.

For any $\xi=\left(\xi_{i}\right)_{i=1}^{k} \in\{-1,1\}^{k}$, let

$$
\Lambda_{\xi}=\left\{\lambda \in \Lambda \mid \xi_{i}\left(P e_{i}\right)^{\prime} \lambda \geq 0, i=1, \ldots, k\right\},
$$

where $\left\{e_{1}, \ldots, e_{k}\right\}$ is the canonical basis of $\mathbb{R}^{k}$. Then, one easily checks that

$$
\Lambda=\bigcup_{\xi \in\{-1,1\}^{k}} \Lambda_{\xi}
$$

In fact, observe that, for any $\lambda \in \Lambda$, if we take $\xi^{\lambda} \in\{-1,1\}^{k}$ such that $\xi_{i}^{\lambda}$ coincides with the sign of the $i$-th coordinate of $P^{\prime} \lambda$ if such a coordinate is nonzero, we have that $\lambda \in \Lambda_{\xi \lambda}$.

Now, for each $\xi \in\{-1,1\}^{k}$ such that $\Lambda_{\xi} \neq \emptyset$, define,

$$
m_{\bar{b}}^{\xi}(x):=\sup _{\lambda \in \Lambda_{\xi}} \frac{(-P \bar{b}-q+A x)^{\prime} \lambda}{\left\|P^{\prime} \lambda\right\|_{1}} \text {, for each } x \in \mathbb{R}^{n} .
$$


From (18), we have

$$
m_{\bar{b}}(x)=\max _{\xi \in\{-1,1\}^{k}, \Lambda_{\xi} \neq \emptyset} m_{\bar{b}}^{\xi}(x), \text { for all } x \in \mathbb{R}^{n} .
$$

Since

$$
\left\|P^{\prime} \lambda\right\|_{1}=(P \xi)^{\prime} \lambda, \text { provided that } \lambda \in \Lambda_{\xi},
$$

for any $x \in \mathbb{R}^{n}$ and $\xi \in\{-1,1\}^{k}$ with $\Lambda_{\xi} \neq \emptyset, m_{\bar{b}}^{\xi}(x)$ coincides with the optimal value of a linear fractional problem in the variable $\lambda$ over the polyhedron $\Lambda_{\xi}$; i.e.,

$$
m_{\bar{b}}^{\xi}(x)=\sup _{\lambda \in \Lambda_{\xi}} \frac{(-P \bar{b}-q+A x)^{\prime} \lambda}{(P \xi)^{\prime} \lambda}
$$

in fact, one easily checks that the supremum in (22) is attained, as a consequence of the compactness of each $\Lambda_{\xi}$ and the assumption that the denominator in (22) is always non zero. Hence, it is known that this optimal value is always attained at some extreme point of $\Lambda_{\xi}$ (see,e.g.,[3]). Denoting by extr $\Lambda_{\xi}$ the set of such extreme points, we have

$$
m_{\bar{b}}^{\xi}(x)=\max _{\lambda \in \operatorname{extr} \Lambda_{\xi}} \frac{(-P \bar{b}-q+A x)^{\prime} \lambda}{(P \xi)^{\prime} \lambda}, \xi \in\{-1,1\}^{k}, \Lambda \xi \neq \emptyset, x \in \mathbb{R}^{n} .
$$

Hence, just take

$$
\widetilde{\Lambda}=\bigcup_{\xi \in\{-1,1\}^{k}, \Lambda_{\xi} \neq \varnothing} \operatorname{extr} \Lambda_{\xi},
$$

and from (20), (21) and (23) we conclude

$$
f_{\bar{b}}(x)=\max _{\lambda \in \widetilde{\Lambda}} \frac{(-P \bar{b}-q+A x)^{\prime} \lambda}{\left\|P^{\prime} \lambda\right\|_{1}}, x \in \mathbb{R}^{n} .
$$

Finally, the second part of the statement comes from Remark 3.

Remark 6 The procedure for finding the finite subset $\tilde{\Lambda} \subset \Lambda$ used in the representation of $m_{\bar{b}}$ as a polyhedral function is implicit in the proof of the previous proposition. The following example is intended to illustrate this procedure.

Example 5 Consider the system in $\mathbb{R}$ (endowed with the absolute value)

$$
\left\{\begin{array}{r}
x \leq b_{1}+b_{2}, t=1 \\
-x \leq-b_{1}+b_{2}, t=2
\end{array}\right\}
$$

In this case $P e_{1}=(1,-1)^{\prime}, P e_{2}=(1,1)^{\prime}, \Lambda=\left\{\lambda \in \mathbb{R}_{+}^{2} \mid \lambda_{1}+\lambda_{2}=1\right\}$, and

$$
\begin{aligned}
\operatorname{extr} \Lambda_{(1,1)} & =\operatorname{extr}\left\{\lambda \in \Lambda \mid \lambda_{1}-\lambda_{2} \geq 0, \lambda_{1}+\lambda_{2} \geq 0\right\}=\left\{\left(\frac{1}{2}, \frac{1}{2}\right)^{\prime},(1,0)^{\prime}\right\}, \\
\operatorname{extr} \Lambda_{(-1,1)} & =\operatorname{extr}\left\{\lambda \in \Lambda \mid-\lambda_{1}+\lambda_{2} \geq 0, \lambda_{1}+\lambda_{2} \geq 0\right\}=\left\{\left(\frac{1}{2}, \frac{1}{2}\right)^{\prime},(0,1)^{\prime}\right\}, \\
\operatorname{extr} \Lambda_{(1,-1)} & =\operatorname{extr} \Lambda_{(-1,-1)}=\emptyset .
\end{aligned}
$$




$$
\begin{aligned}
& \text { Hence } \widetilde{\Lambda}=\left\{(1,0)^{\prime},\left(\frac{1}{2}, \frac{1}{2}\right)^{\prime},(0,1)^{\prime}\right\} \text {. Take } \bar{b}=0_{2} \text {. Then } \\
& \begin{aligned}
f_{\bar{b}}(x) & =\max \left\{\frac{\left(\begin{array}{c}
-x \\
x
\end{array}\right)^{\prime}\left(\begin{array}{l}
1 \\
0
\end{array}\right)}{\left\|\left(\begin{array}{l}
1 \\
1
\end{array}\right)\right\|_{1}}, \frac{\left(\begin{array}{c}
-x \\
x
\end{array}\right)^{\prime}\left(\begin{array}{c}
1 / 2 \\
1 / 2
\end{array}\right)}{\left\|\left(\begin{array}{l}
0 \\
1
\end{array}\right)\right\|_{1}}, \frac{\left(\begin{array}{c}
-x \\
x
\end{array}\right)^{\prime}\left(\begin{array}{l}
0 \\
1
\end{array}\right)}{\left\|\left(\begin{array}{c}
-1 \\
1
\end{array}\right)\right\|_{1}}\right\} \\
& =\max \left\{\frac{-x}{2}, 0, \frac{x}{2}\right\}=\frac{1}{2}|x|, x \in \mathbb{R} .
\end{aligned}
\end{aligned}
$$

Therefore, taking $\bar{x}=0, \partial^{>} f_{\bar{b}}(\bar{x})=\left\{-\frac{1}{2}, \frac{1}{2}\right\}$ and

$$
\operatorname{clm} \mathcal{F}(\bar{b}, \bar{x})=d\left(0, \partial^{>} f_{\bar{b}}(\bar{x})\right)^{-1}=2 .
$$

\subsection{Structured vs Arbitrary RHS Perturbations}

Given $(\bar{b}, \bar{x}) \in \operatorname{gph} \mathcal{F}$, we define the set of active indices and its subsets of unperturbable/perturbable active indices (for $\bar{b}$ ) at $\bar{x}$ as, respectively,

$$
\begin{aligned}
T_{\bar{b}}(\bar{x}) & :=\left\{t \in T: a_{t}^{\prime} \bar{x}=q_{t}+p_{t}^{\prime} \bar{b}\right\}, \\
T_{\bar{b}}^{0}(\bar{x}) & :=\left\{t \in T_{\bar{b}}(\bar{x}): p_{t}=0_{k}\right\}, \\
T_{\bar{b}}^{1}(\bar{x}) & :=T_{\bar{b}}(\bar{x}) \backslash T_{\bar{b}}^{0}(\bar{x}) .
\end{aligned}
$$

In order to compare the following proposition with the result of Lemma 1, we consider the submatrices of $A$, denoted by $\bar{A}, A_{0}$ and $A_{1}$, formed by the rows of $A$ which are indexed by $T_{\bar{b}}(\bar{x}), T_{\bar{b}}^{0}(\bar{x})$ and $T_{\bar{b}}^{1}(\bar{x})$; i.e.,

$$
\bar{A}:=\left(a_{t}^{\prime}\right)_{t \in T_{\bar{b}}(\bar{x})}, A_{0}=\left(a_{t}^{\prime}\right)_{t \in T_{\bar{b}}^{0}(\bar{x})} \text { and } A_{1}=\left(a_{t}^{\prime}\right)_{t \in T_{\bar{b}}^{1}(\bar{x})} .
$$

In the same way, $\bar{P}:=\left(p_{t}^{\prime}\right)_{t \in T_{\bar{b}}(\bar{x})}, P_{1}:=\left(p_{t}^{\prime}\right)_{t \in T_{\bar{b}}^{1}(\bar{x})}$, and

$$
\bar{\Lambda}:=\left\{\lambda \in \mathbb{R}_{+}^{\left|T_{\bar{b}}(\bar{x})\right|}: \sum_{t=1}^{\left|T_{\bar{b}}(\bar{x})\right|} \lambda_{t}=1\right\}, \Lambda_{1}:=\left\{\lambda \in \mathbb{R}_{+}^{\left|T_{\bar{b}}^{1}(\bar{x})\right|}: \sum_{t=1}^{\left|T_{\bar{b}}^{1}(\bar{x})\right|} \lambda_{t}=1\right\} .
$$

where $|E|$ stands for the cardinality of the finite set $E$.

Proposition 3 Given $(\bar{b}, \bar{x}) \in \operatorname{gph} \mathcal{F}$, there exists a neighborhood $W$ of $\bar{x}$ such that

$$
d\left(\bar{b}, \mathcal{F}^{-1}(x)\right)=\max _{\lambda \in \Lambda_{1}} \frac{\left[\left(A_{1}(x-\bar{x})\right)^{\prime} \lambda\right]_{+}}{\left\|P_{1}^{\prime} \lambda\right\|_{1}},
$$

whenever $x \in W \cap \operatorname{dom} \mathcal{F}^{-1}$, under the convention that the maximum over the empty set equals zero.

Proof First, let us prove the existence of a neighborhood $W$ of $\bar{x}$ such that

$$
d\left(\bar{b}, \mathcal{F}^{-1}(x)\right)=d\left(\bar{b},\left\{b \in \mathbb{R}^{k}:-p_{t}^{\prime} b \leq q_{t}-a_{t}^{\prime} x, \text { for all } t \in T_{\bar{b}}(\bar{x})\right\}\right),
$$

for all $x \in W \cap \operatorname{dom} \mathcal{F}^{-1}$. To do this, by continuity, take $\varepsilon>0$ such that

$$
-p_{t}^{\prime} b<q_{t}-a_{t}^{\prime} x \text {, for all } t \notin T_{\bar{b}}(\bar{x}),
$$


whenever $\|x-\bar{x}\|<\varepsilon$ and $\|b-\bar{b}\|_{\infty}<\varepsilon$. From a classical result by Hoffman [18], we derive the (Berge) lower semicontinuity -indeed the Lipschitz continuity- of the mapping $\mathcal{G}: \mathbb{R}^{T_{\bar{b}}(\bar{x})} \rightrightarrows \mathbb{R}^{k}$ given by

$$
\mathcal{G}(d):=\left\{b \in \mathbb{R}^{k}:-p_{t}^{\prime} b \leq d_{t}, t \in T_{\bar{b}}(\bar{x})\right\},
$$

relative to its domain, at $\bar{d}:=\left(q_{t}-a_{t}^{\prime} \bar{x}\right)_{t \in T_{\bar{b}}(\bar{x})}$. Accordingly, since $\bar{b} \in \mathcal{G}(\bar{d})$, there exists $\delta \in] 0, \varepsilon[$ such that

$$
\|x-\bar{x}\|<\delta \Rightarrow \mathcal{G}\left(\left(q_{t}-a_{t}^{\prime} x\right)_{t \in T_{\bar{b}}(\bar{x})}\right) \cap\left\{b \in \mathbb{R}^{k}:\|b-\bar{b}\|_{\infty}<\varepsilon\right\} \neq \emptyset,
$$

provided that $\mathcal{G}\left(\left(q_{t}-a_{t}^{\prime} x\right)_{t \in T_{\bar{b}}(\bar{x})}\right) \neq \emptyset$; in particular when $x \in \operatorname{dom} \mathcal{F}^{-1}$.

Define $W:=\left\{x \in \mathbb{R}^{n}:\|x-\bar{x}\|<\delta\right\}$, pick any $x \in W \cap \operatorname{dom} \mathcal{F}^{-1}$ and let $\widehat{b} \in \mathbb{R}^{k}$ be such that

$$
\|\widehat{b}-\bar{b}\|_{\infty}=d\left(\bar{b}, \mathcal{G}\left(\left(q_{t}-a_{t}^{\prime} x\right)_{t \in T_{\bar{b}}(\bar{x})}\right)\right) .
$$

Then, (28) entails $\|\widehat{b}-\bar{b}\|_{\infty}<\varepsilon$, and therefore, $-p_{t}^{\prime} \widehat{b}<q_{t}-a_{t}^{\prime} x$, for all $t \notin T_{\bar{b}}(\bar{x})$ because of (27). Hence, $\widehat{b} \in \mathcal{F}^{-1}(x) \subset \mathcal{G}\left(\left(q_{t}-a_{t}^{\prime} x\right)_{t \in T_{\bar{b}}(\bar{x})}\right)$, which implies that

$$
d\left(\bar{b}, \mathcal{F}^{-1}(x)\right)=\|\widehat{b}-\bar{b}\|_{\infty} .
$$

In other words, (26) holds for all $x \in W \cap \operatorname{dom} \mathcal{F}^{-1}$. Now, in a similar way to the proof of Lemma 1 (i.e., appealing to [12, Lemma 1]), given $x \in W \cap \operatorname{dom} \mathcal{F}^{-1}$, we can write

$$
d\left(\bar{b}, \mathcal{F}^{-1}(x)\right)=\max _{\lambda \in \bar{\Lambda}} \frac{\left[(\bar{A}(x-\bar{x}))^{\prime} \lambda\right]_{+}}{\left\|\bar{P}^{\prime} \lambda\right\|_{1}} .
$$

If for any $\lambda \in \bar{\Lambda}$ we denote $\lambda^{i}:=\left(\lambda_{t}\right)_{t \in T \frac{i}{b}(\bar{x})}, i=0,1$, it is obvious that $\bar{P}^{\prime} \lambda=P_{1}^{\prime} \lambda^{1}$. Moreover,

$$
\begin{aligned}
\frac{\left[(\bar{A}(x-\bar{x}))^{\prime} \lambda\right]_{+}}{\left\|\bar{P}^{\prime} \lambda\right\|_{1}} & =\frac{\left[\left(A_{1}(x-\bar{x})\right)^{\prime} \lambda^{1}+\left(A_{0}(x-\bar{x})\right)^{\prime} \lambda^{0}\right]_{+}}{\left\|P_{1}^{\prime} \lambda^{1}\right\|_{1}} \\
& \leq \frac{\left[\left(A_{1}(x-\bar{x})\right)^{\prime} \lambda^{1}\right]_{+}}{\left\|P_{1}^{\prime} \lambda^{1}\right\|_{1}}=\frac{\left[\left(A_{1}(x-\bar{x})\right)^{\prime} \tilde{\lambda}^{1}\right]_{+}}{\left\|P_{1}^{\prime} \tilde{\lambda}^{1}\right\|_{1}}
\end{aligned}
$$

where $\tilde{\lambda}^{1}:=\left(\sum_{t \in T \frac{1}{b}(\bar{x})} \lambda_{t}^{1}\right)^{-1} \lambda^{1}$ and we used the inequality $\left(A_{0}(x-\bar{x})\right)^{\prime} \lambda^{0} \leq 0$ which comes from $x \in \operatorname{dom} \mathcal{F}^{-1}$. Therefore, one immediately derives (25).

Now we are ready to provide lower and upper estimates of $\operatorname{clm} \mathcal{F}(\bar{b}, \bar{x})$. Our estimates are in terms of the calmness modulus of the feasible set mapping $\mathcal{F}_{T^{1}, T^{0}}: \mathbb{R}^{T^{1}} \rightrightarrows \mathbb{R}^{n}$ given by - see (10)-

$$
\mathcal{F}_{T^{1}, T^{0}}(\xi):=\left\{x \in \mathbb{R}^{n}: a_{t}^{\prime} x \leq \xi_{t}, t \in T^{1} ; a_{t}^{\prime} x \leq q_{t}, \text { if } t \in T^{0}\right\},
$$

where $\xi=\left(\xi_{t}\right)_{t \in T^{1}} \in \mathbb{R}^{T^{1}}$ and, similarly to (24), we use the notation

$$
T^{1}:=\left\{t \in T: p_{t} \neq 0_{k}\right\}, T^{0}:=T \backslash T^{1} .
$$


Specifically, given $(\bar{b}, \bar{x}) \in \operatorname{gph} \mathcal{F}$, the following theorem estimates $\operatorname{clm} \mathcal{F}(\bar{b}, \bar{x})$ in terms of $\operatorname{clm} \mathcal{F}_{T^{1}, T^{0}}\left(b^{1}, \bar{x}\right)$, where

$$
b^{1}:=\left(q_{t}+p_{t}^{\prime} \bar{b}\right)_{t \in T^{1}} \in \mathbb{R}^{T^{1}} .
$$

In this way, the theorem estimates the calmness modulus of the feasible set mapping in the context of linear systems under structured perturbations in terms of the naturally arising mapping in the setting of non-structured perturbations, for which the reader can find a vast literature.

At this moment we point out the fact that $\operatorname{clm} \mathcal{F}_{T^{1}, T^{0}}\left(b^{1}, \bar{x}\right)$ was analyzed in previous works; indeed, it may be directly computed from Theorem 2 with the roles of $I_{\bar{b}}(\bar{x})$ and $U(\bar{x})$ being respectively played by $T_{\bar{b}}^{1}(\bar{x})$ and $T_{\bar{b}}^{0}(\bar{x})$.

Theorem 3 Let $(\bar{b}, \bar{x}) \in \operatorname{gph} \mathcal{F}$. We have

(i) If $T_{\bar{b}}^{1}(\bar{x})=\emptyset$, then $\operatorname{clm} \mathcal{F}(\bar{b}, \bar{x})=0$;

(ii) If $T_{\bar{b}}^{1}(\bar{x}) \neq \emptyset$, then

$$
\alpha \operatorname{clm} \mathcal{F}_{T^{1}, T^{0}}\left(b^{1}, \bar{x}\right) \leq \operatorname{clm} \mathcal{F}(\bar{b}, \bar{x}) \leq \beta \operatorname{clm} \mathcal{F}_{T^{1}, T^{0}}\left(b^{1}, \bar{x}\right),
$$

where $\alpha$ and $\beta$ stand respectively for the minimum and the maximum of $\left\{\|v\|_{1}: v \in \operatorname{conv}\left\{p_{t}: t \in T_{\bar{b}}^{1}(\bar{x})\right\}\right\}$.

If, in addition, $P_{1}^{\prime} \lambda=0_{k}$ implies $A_{1}^{\prime} \lambda=0_{n}\left(\lambda \in \mathbb{R}^{\left|T_{\bar{b}}^{1}(\bar{x})\right|}\right)$, then $\alpha$ in (31) may be replaced with $\min \left\{\|v\|_{1}: v \in\right.$ end conv $\left.\left\{p_{t}: t \in T_{\bar{b}}^{1}(\bar{x})\right\}\right\}$.

Proof (i) The reader can easily check by continuity arguments that $T_{\bar{b}}(\bar{x})=T_{\bar{b}}^{0}(\bar{x})$ implies that $\mathcal{F}(b) \cap W_{0}=\mathcal{F}(\bar{b}) \cap W_{0}$ for a certain neighborhood $W_{0}$ of $\bar{x}$ and all $b$ in a certain neighborhood $V_{0}$ of $\bar{b}$, and in this situation clearly $\operatorname{clm} \mathcal{F}(\bar{b}, \bar{x})=0$.

(ii) Assume the nontrivial case $\alpha>0$. From (7) we can write

$$
\operatorname{clm} \mathcal{F}(\bar{b}, \bar{x})=\limsup _{x \rightarrow \bar{x}, x \in \operatorname{dom} \mathcal{F}^{-1}} \frac{d(x, \mathcal{F}(\bar{b}))}{d\left(\bar{b}, \mathcal{F}^{-1}(x)\right)},
$$

since the lim sup may be confined to those $x \in \mathbb{R}^{n}$ such that $d\left(\bar{b}, \mathcal{F}^{-1}(x)\right)<+\infty$, i.e. $x \in \operatorname{dom} \mathcal{F}^{-1}$.

First, observe that, from (30),

$$
\mathcal{F}(\bar{b})=\mathcal{F}_{T^{1}, T^{0}}\left(b^{1}\right) .
$$

Next, let us estimate $d\left(\bar{b}, \mathcal{F}^{-1}(x)\right)$ in terms of $d\left(b^{1}, \mathcal{F}_{T^{1}, T^{0}}^{-1}(x)\right)$, provided that $x \in$ $\operatorname{dom} \mathcal{F}^{-1}$ is close enough to $\bar{x}$. Note that $\operatorname{dom} \mathcal{F}^{-1} \subset \operatorname{dom} \mathcal{F}_{T^{1}, T^{0}}^{-1}$ since $b \in \mathcal{F}^{-1}(x)$ entails $\left(q_{t}+p_{t}^{\prime} b\right)_{t \in T^{1}} \in \mathcal{F}_{T^{1}, T^{0}}^{-1}(x)$.

Take $W$ as in Proposition 3 and consider $\alpha$ and $\beta$ as in (ii). Then, for each $x \in W \cap$ $\operatorname{dom} \mathcal{F}^{-1}$, from (25) we deduce

$$
\beta^{-1} \max _{\lambda \in \Lambda_{1}}\left[\left(A_{1}(x-\bar{x})\right)^{\prime} \lambda\right]_{+} \leq d\left(\bar{b}, \mathcal{F}^{-1}(x)\right) \leq \alpha^{-1} \max _{\lambda \in \Lambda_{1}}\left[\left(A_{1}(x-\bar{x})\right)^{\prime} \lambda\right]_{+} .
$$


Moreover, since $a_{t}^{\prime} \bar{x}=q_{t}+p_{t}^{\prime} \bar{b}=b_{t}^{1}$ for $t \in T_{\bar{b}}^{1}(\bar{x})$, one easily sees that

$$
\begin{aligned}
\max _{\lambda \in \Lambda_{1}}\left[\left(A_{1}(x-\bar{x})\right)^{\prime} \lambda\right]_{+} & =\max _{t \in T_{\bar{b}}^{1}(\bar{x})}\left[a_{t}^{\prime}(x-\bar{x})\right]_{+}=\max _{t \in T_{\bar{b}}^{1}(\bar{x})}\left[a_{t}^{\prime} x-b_{t}^{1}\right]_{+} \\
& =d\left(b^{1}, \mathcal{F}_{T^{1}, T^{0}}^{-1}(x)\right),
\end{aligned}
$$

whenever $x \in \operatorname{dom} \mathcal{F}_{T^{1}, T^{0}}^{-1}$ is close enough to $\bar{x}$ (to guarantee $a_{t}^{\prime} x<b_{t}^{1}$, for $t \in T^{1} \backslash T_{\bar{b}}^{1}(\bar{x})$ ), say $x \in W_{1}$ for a certain neighborhood $W_{1} \subset W$ of $\bar{x}$. Consequently, for $x \in W_{1} \cap \operatorname{dom} \mathcal{F}^{-1}$,

$$
\beta^{-1} d\left(b^{1}, \mathcal{F}_{T^{1}, T^{0}}^{-1}(x)\right) \leq d\left(\bar{b}, \mathcal{F}^{-1}(x)\right) \leq \alpha^{-1} d\left(b^{1}, \mathcal{F}_{T^{1}, T^{0}}^{-1}(x)\right) .
$$

Thus, (32) and (33) yield

$$
\frac{d\left(x, \mathcal{F}_{T^{1}, T^{0}}\left(b^{1}\right)\right)}{\alpha^{-1} d\left(b^{1}, \mathcal{F}_{T^{1}, T^{0}}^{-1}(x)\right)} \leq \frac{d(x, \mathcal{F}(\bar{b}))}{d\left(\bar{b}, \mathcal{F}^{-1}(x)\right)} \leq \frac{d\left(x, \mathcal{F}_{T^{1}, T^{0}}\left(b^{1}\right)\right)}{\beta^{-1} d\left(b^{1}, \mathcal{F}_{T^{1}, T^{0}}^{-1}(x)\right)},
$$

whenever $x \in W_{1} \cap \operatorname{dom} \mathcal{F}^{-1}$. Finally, letting $x \rightarrow \bar{x}$ we obtain (31).

In order to improve the choice of $\alpha$ under the additional hypothesis, it is enough to see that, for $x \in W \cap \operatorname{dom} \mathcal{F}^{-1}$, the maximum in (25), assumed to be attained at a certain $\widehat{\lambda} \in \Lambda_{1}$, is also attained at some $\tilde{\lambda} \in \Lambda_{1}$ such that $P_{1}^{\prime} \tilde{\lambda} \in$ end conv $\left\{p_{t}, t \in T_{\bar{b}}^{1}(\bar{x})\right\}$. Let $\mu \geq 1$ be such that $\mu P_{1}^{\hat{\lambda}} \in$ end conv $\left\{p_{t}, t \in T_{\bar{b}}^{1}(\bar{x})\right\}$. By the compactness of conv $\left\{p_{t}, t \in T_{\bar{b}}^{1}(\bar{x})\right\}$, take $\tilde{\lambda} \in \Lambda_{1}$ such that $\mu P_{1}^{\prime} \widehat{\lambda}=P_{1}^{\prime} \tilde{\lambda}$; i.e., $P_{1}^{\prime}(\mu \widehat{\lambda}-\tilde{\lambda})=0_{k}$. Hence, our assumption entails $A_{1}^{\prime}(\mu \widehat{\lambda}-\tilde{\lambda})=0_{n}$, yielding

$$
\frac{\left[\left(A_{1}(x-\bar{x})\right)^{\prime} \widehat{\lambda}\right]_{+}}{\left\|P_{1}^{\prime} \hat{\lambda}\right\|_{1}}=\frac{\left[\left(A_{1}(x-\bar{x})\right)^{\prime}(\mu \widehat{\lambda})\right]_{+}}{\left\|P_{1}^{\prime}(\mu \widehat{\lambda})\right\|_{1}}=\frac{\left[\left(A_{1}(x-\bar{x})\right)^{\prime} \tilde{\lambda}\right]_{+}}{\left\|P_{1}^{\prime} \tilde{\lambda}\right\|_{1}} .
$$

Remark 7 Obviously, both inequalities in (31) become equalities when $\alpha=\beta$. Otherwise, any of them may either be attained or be strict. On the one hand, in Example 5 $\operatorname{clm} \mathcal{F}(\bar{b}, \bar{x})=2$ while one easily checks that $\operatorname{clm} \mathcal{F}_{T^{1}, T^{0}}\left(b^{1}, \bar{x}\right)=1$ (in that case, $\bar{x}=0$, $\bar{b}=b^{1}=0_{2}$ ). Moreover, $\left\{p_{t}: t \in T_{\bar{b}}^{1}(\bar{x})\right\}=\left\{(1,1)^{\prime},(-1,1)^{\prime}\right\}$ and then $\alpha=1$ (for both possible choices) and $\beta=2$. So, in that example, the first inequality in (31) is strict while the second holds as an equality.

On the other hand, if we consider the parametrized system in $\mathbb{R}\left\{x \leq b_{1}, x \leq 2 b_{2}\right\}$, one easily sees that, taking $\bar{x}=0, \bar{b}=b^{1}=0_{2}$, we have $\operatorname{clm} \mathcal{F}_{T^{1}, T^{0}}\left(b^{1}, \bar{x}\right)=\operatorname{clm} \mathcal{F}(\bar{b}, \bar{x})=1$. Moreover $\alpha=1$ (again for both choices) and $\beta=2$. So, here, the first inequality in (31) holds as equality and the second is strict.

Corollary 1 In the cases of Examples 1 and 3, for any $(\bar{b}, \bar{x}) \in \operatorname{gph} \mathcal{F}$ with $T_{\bar{b}}^{1}(\bar{x}) \neq \varnothing$ we have

$$
\operatorname{clm} \mathcal{F}(\bar{b}, \bar{x})=\operatorname{clm} \mathcal{F}_{T^{1}, T^{0}}\left(b^{1}, \bar{x}\right) .
$$

Proof First, it can be immediately checked that the additional hypothesis of Theorem $3(i i)$ is fulfilled for Examples 1 and 3. So, we only have to prove that in the referred 
cases one has $\alpha=\beta=1$, where $\alpha$ is chosen as the minimum of $\|v\|_{1}$ with $v \in$ end conv $\left\{p_{t}: t \in T_{\bar{b}}^{1}(\bar{x})\right\}$.

In the case of Example 1 all rows of $P$ have exactly one 1 with the remaining entries being 0 . In other words, for all $t \in T=\{1, \ldots, m\}$ we have that each $p_{t}$ is an extreme point of the standard simplex of $\mathbb{R}^{k}$ (some of them possibly repeated). In this case it is clear that $\|v\|_{1}=1$ for all $v \in \operatorname{conv}\left\{p_{t}, t \in T_{\bar{b}}(\bar{x})\right\}$, and we are done.

In the case of Example 3 we immediately see by looking at matrix $P$ that, for all $t \in T=\{1, \ldots, m\}$, each $p_{t}$ is either an extreme point of the $l_{1}$ ball of $\mathbb{R}^{k}$ (some of them possibly repeated) or the origin, $0_{k}$. In this case it is clear that $\|v\|_{1} \leq 1$ for all $v \in \operatorname{conv}\left\{p_{t}, t \in T_{\bar{b}}(\bar{x})\right\}$, but we can easily conclude that $\|v\|_{1}=1$ for all $v \in$ end conv $\left\{p_{t}, t \in T_{\bar{b}}(\bar{x})\right\}$.

Now we are ready to compute the calmness modulus of the feasible set mapping for systems of equalities and inequalities given in Example 3. From the notational point of view, according to the notation in (10), it is convenient to make explicit a partition $\{I, E, U\}$ of the index set $T=\{1, \ldots, m\}$ and to define $\mathcal{F}: \mathbb{R}^{I \cup E} \rightrightarrows \mathbb{R}^{n}$ by

$$
\mathcal{F}(b):=\left\{a_{t}^{\prime} x \leq b_{t}, t \in I ; a_{t}^{\prime} x=b_{t}, t \in E ; a_{t}^{\prime} x \leq b_{t}^{0}, t \in U\right\},
$$

where $I, E$ (after 'equalities'), and $U$ are disjoint finite sets, $b:=\left(b_{t}\right)_{t \in I \cup E} \in \mathbb{R}^{I \cup E}$ is the parameter to be perturbed around a nominal value $\bar{b}:=\left(\bar{b}_{t}\right)_{t \in I \cup E}$, and $\left(b_{t}^{0}\right)_{t \in U}$ remains fixed. Note that this framework also allows for unperturbed equalities by just splitting each of them into two inequalities (see Remark 4).

For $\bar{x} \in \mathcal{F}(\bar{b})$, and recalling the notation (12), let $\mathcal{D}(\bar{x})$ stand for the family of all $\left(D_{1}, D_{2}^{+}, D_{2}^{-}, D_{3}\right)$ with $D_{1} \subset I_{\bar{b}}(\bar{x}) ; D_{2}^{+}, D_{2}^{-} \subset E$ with $D_{2}^{+} \cap D_{2}^{-}=\emptyset$; and $D_{3} \subset U(\bar{x})$ such that the system

$$
\left\{\begin{array}{ll}
a_{t}^{\prime} d=1, & t \in D_{1}, \\
a_{t}^{\prime} d<1, & t \in I_{\bar{b}}(\bar{x}) \backslash D_{1}, \\
a_{t}^{\prime} d=1, & t \in D_{2}^{+}, \\
a_{t}^{\prime} d=-1, & t \in D_{2}^{-}, \\
\left|a_{t}^{\prime} d\right|<1, & t \in E \backslash\left(D_{2}^{+} \cup D_{2}^{-}\right), \\
a_{t}^{\prime} d=0, & t \in D_{3}, \\
a_{t}^{\prime} d<0, & t \in U(\bar{x}) \backslash D_{3},
\end{array}\right\}
$$

is consistent (in the variable $d \in \mathbb{R}^{n}$ ).

The following result comes directly from Corollary 1 and extends the last part of Theorem 2 (see also [11, Formula (16) and Theorem 4]).

Corollary 2 Let $\mathcal{F}$ be as in (34). For $(\bar{b}, \bar{x}) \in \operatorname{gph} \mathcal{F}$ we have

$$
\operatorname{clm} \mathcal{F}(\bar{b}, \bar{x})=\left(\min _{\left(D_{1}, D_{2}^{+}, D_{2}^{-}, D_{3}\right) \in \mathcal{D}(\bar{x})} \delta\left(D_{1}, D_{2}^{+}, D_{2}^{-}, D_{3}\right)\right)^{-1},
$$

where $\delta\left(D_{1}, D_{2}^{+}, D_{2}^{-}, D_{3}\right)$ is defined as

$$
d_{*}\left(0_{n}, \text { conv }\left\{a_{t}, t \in D_{1} \cup D_{2}^{+} ;-a_{t}, t \in D_{2}^{-}\right\}+\text {cone }\left\{a_{t}, t \in D_{3}\right\}\right) .
$$




\section{Application to the Convergence of the Primal-Dual Path-Following Method}

Let us consider to the primal-dual pair of LP problems:

$$
\begin{aligned}
& \text { (P) } \min c^{\prime} x \\
& \text { s.t. } A x=b \text {, } \\
& x \geq 0_{n},
\end{aligned}
$$

where $c \in \mathbb{R}^{n}, A \in \mathbb{R}^{m \times n}$ and $b \in \mathbb{R}^{m}$ are given data, and $x \in \mathbb{R}^{n}$ and $(y, z) \in \mathbb{R}^{m} \times \mathbb{R}^{n}$ represent, respectively, the vectors of primal and dual variables. In the sequel $S(P) \subset \mathbb{R}^{n}$ and $S(D) \subset \mathbb{R}^{m} \times \mathbb{R}^{n}$ stand for the optimal sets of $(P)$ and $(D)$, respectively.

Associated with problem $(P)$, for each scalar $\mu>0$ we consider the well-known logarithmic barrier problem,

$$
\begin{aligned}
\left(P_{\mu}\right) \min & c^{\prime} x-\mu \sum_{i=1}^{n} \log x_{i} \\
\text { s.t. } & A x=b, \\
& x>0_{n} .
\end{aligned}
$$

The set of optimal solutions of $\left(P_{\mu}\right)$ is known to coincide with the set of $x \in \mathbb{R}^{n}$ such that there exists $(y, z) \in \mathbb{R}^{m} \times \mathbb{R}^{n}$ verifying the following nonlinear system coming from the Karush-Kuhn-Tucker optimality conditions:

$$
\left\{\begin{array}{l}
F_{\mu}(x, y, z)=0_{2 n+m}, \\
x, z \geq 0_{n},
\end{array}\right.
$$

where $F_{\mu}: \mathbb{R}^{n} \times \mathbb{R}^{m} \times \mathbb{R}^{n} \rightarrow \mathbb{R}^{n} \times \mathbb{R}^{m} \times \mathbb{R}^{n}$ is given by

$$
F_{\mu}(x, y, z)=\left(A^{\prime} y+z-c, A x-b,\left(x_{i} z_{i}-\mu\right)_{i=1, \ldots, n}\right) .
$$

Remark 8 If we denote by $\Lambda$ the set of solutions of system (36) in the case when $\mu=0$, a standard argument of LP yields

$$
\Lambda=S(P) \times D(P) ;
$$

i.e., $\Lambda$ is nothing else but the set of primal-dual solutions of (35).

For completeness purposes, and in order to integrate the current application in the literature, we recall that [27, Proposition 8.1] establishes that problem $\left(P_{\mu}\right)$ is either unbounded for every $\mu>0$ or has a unique optimal solution, denoted by $x(\mu)$, for every $\mu>0$. In this way, if $\left(P_{\mu}\right)$ is solvable for some positive value $\mu$, then $x(\cdot): \mu \mapsto x(\mu)$ determines a unique path, yielding an interior point method with $x(\mu)>0_{n}$ for all $\mu>0$. Assumptions $2.1(\mathrm{a}, \mathrm{b}$, and $\mathrm{c})$ in [28], which are equivalent to the simultaneous fulfilment of the Slater constraint qualification and the fact that $S(P)$ is non-empty and bounded (see [6, Sec. 4] for details), ensure the existence of such $x(\mu)$ for each $\mu>0$. Moreover, [27, Proposition 8.2] establishes the continuity of $x(\cdot)$ and the existence of $x^{0}:=\lim _{\mu \rightarrow 0} x(\mu)$, which is an optimal solution of our LP problem $(P)$. The reader is addressed to [4, Chapter 9] for details and additional references on this subject. See also [20] for the first interior point algorithm. If we denote by $(x(\mu), y(\mu), z(\mu))$ the complete solution of the KKT system (36), the path $\{(x(\mu), y(\mu), z(\mu)) ; \mu>0\}$ is usually referred to as the central path associated with LP problem $(P)$ (see the complete description in [28, Section 2]). The reader is 
addressed to [6, Section 4] for an application of calmness constants in the context of linear inequality systems to the computation of constants $\widetilde{\kappa} \geq 0$ satisfying

$$
d((x(\mu), y(\mu), z(\mu)), \Lambda)) \leq \widetilde{\kappa} \mu \text { for } \mu>0 \text { sufficiently small. }
$$

Now we are going to apply the classical Newton method to approach possible solutions of the KKT system (36). In this way, we are dealing with the so-called primal-dual pathfollowing method. One can find in the literature particular implementations of this generic scheme, such as the pioneering works [28, 32]. See also [1] for a different implementation, coming from a specific reduced KKT system. The reader is addressed to $[16,30]$ for comprehensive surveys on the field of interior point methods.

Here we consider a particular version of the primal-dual path-following method. Specifically, at each iteration $r \in \mathbb{N}$ we consider a nonnegative scalar $\mu_{r}$ in such a way that $\mu_{r} \rightarrow 0$ as $r \rightarrow \infty$. Starting from any initial element $w^{1}=\left(x^{1}, y^{1}, z^{1}\right) \in \mathbb{R}_{+}^{n} \times \mathbb{R}^{m} \times \mathbb{R}_{+}^{n}$, we generate a sequence $\left\{w^{r}:=\left(x^{r}, y^{r}, z^{r}\right)\right\}_{r \in \mathbb{N}} \subset \mathbb{R}^{n} \times \mathbb{R}^{m} \times \mathbb{R}^{n}$ through the following recursive scheme:

Iteration $r$ : Take $w^{r+1}:=\left(x^{r+1}, y^{r+1}, z^{r+1}\right)$ as a solution value of the variable $w=$ $(x, y, z) \in \mathbb{R}^{n} \times \mathbb{R}^{m} \times \mathbb{R}^{n}$ for the linear system (coming from applying one iteration of the Newton method to system (36) and adding a nonnegativity condition) with $\mu=\mu_{r}$ :

$$
\left\{\begin{array}{l}
D F_{\mu_{r}}\left(w^{r}\right) w=D F_{\mu_{r}}\left(w^{r}\right) w^{r}-F_{\mu_{r}}\left(w^{r}\right), \\
x, z \geq 0_{n}
\end{array}\right.
$$

where, one easily checks that

$$
\begin{aligned}
D F_{\mu_{r}}\left(w^{r}\right) & =\left(\begin{array}{ccc}
0_{n \times n} & A^{\prime} & I_{n} \\
A & 0_{m \times m} & 0_{m \times n} \\
Z^{r} & 0_{n \times m} & X^{r}
\end{array}\right), \\
F_{\mu_{r}}\left(w^{r}\right) & =\left(A^{\prime} y^{r}+z^{r}-c, A x^{r}-b, X^{r} Z^{r} e-\mu_{r} e\right)
\end{aligned}
$$

where $X^{r}$ and $Z^{r}$ are the diagonal matrices whose diagonal elements are the coordinates of $x^{r}=\left(x_{1}^{r}, \ldots, x_{n}^{r}\right)^{\prime}$ and $z^{r}=\left(z_{1}^{r}, \ldots, z_{n}^{r}\right)^{\prime}$, respectively, and $e:=(1, \ldots, 1)^{\prime}$. The previous system of equalities can be written in the alternative form

$$
\begin{aligned}
Z^{r} x+X^{r} z & =X^{r} Z^{r} e+\mu_{r} e, \\
A x & =b, \\
A^{\prime} y+z & =c .
\end{aligned}
$$

Adopting this notation, assume that $\left\{w^{r}\right\}$ converges to certain $\bar{w}=(\bar{x}, \bar{y}, \bar{z})$. Note that $(\bar{x}, \bar{y}, \bar{z})$ satisfies

$$
\begin{aligned}
\bar{Z} \bar{X} & =0_{n \times n}, \\
A \bar{x} & =b, \\
A^{\prime} \bar{y}+\bar{z} & =c, \\
\bar{x}, \bar{z} & \geq 0_{n},
\end{aligned}
$$

where $\bar{X}$ and $\bar{Z}$ denote the diagonal $n \times n$ matrices whose diagonal elements are the coordinates of $\bar{x}$ and $\bar{z}$ (recall $\mu_{r} \rightarrow 0$ ). So,

$$
(\bar{x}, \bar{y}, \bar{z}) \in \Lambda \text {. }
$$

From now on, for the sake of simplicity, $\|\cdot\|$ denotes the Euclidean norm in any $\mathbb{R}^{l}$, $l \in \mathbb{N}$. Now we define the feasible set mapping $\mathcal{N}:\left(\mathbb{R}^{n},\|\cdot\|_{\infty}\right) \rightrightarrows\left(\mathbb{R}^{n} \times \mathbb{R}^{m} \times \mathbb{R}^{n},\|\cdot\|\right)$ 
assigning to each parameter $\eta \in \mathbb{R}^{n}$ the set

$$
\mathcal{N}(\eta):=\left\{\begin{array}{l|l}
(x, y, z) \in \mathbb{R}^{n} \times \mathbb{R}^{m} \times \mathbb{R}^{n} & \begin{array}{l}
\bar{Z} x+\bar{X} z=\eta, \\
A x=b, \\
A^{\prime} y+z=c, \\
x, z \geq 0_{n},
\end{array}
\end{array}\right\} .
$$

Remark 9 Under the current notation and assumptions, we have that

$$
\mathcal{N}\left(0_{n}\right)=\Lambda \text {. }
$$

In fact, if $(x, y, z) \in \mathcal{N}\left(0_{n}\right)$, then $\bar{Z} x+\bar{X} z=0_{n}$, which implies that $\bar{Z} x=\bar{X} z=0_{n}$. So, $x \in S(P)$ as consequence of the fact that $(x, \bar{y}, \bar{z}) \in \Lambda$, and $(y, z) \in S(D)$ because $(\bar{x}, y, z) \in \Lambda$. Conversely, if $x \in S(P)$ and $(y, z) \in S(D)$, then both $(x, \bar{y}, \bar{z})$ and $(\bar{x}, y, z)$ belong to $\Lambda$, entailing $\bar{Z} x+\bar{X} z=0_{n}$.

In the sequel, for simplicity in the notation, let us assume that $\bar{x}_{i}>0$ for $i=1, \ldots, s$, and $\bar{x}_{i}=0$ for $i=s+1, \ldots, n$. According to the complementarity condition $\left(\bar{x}_{i} \bar{z}_{i}=0\right.$, for all $i$ ), we have $\bar{z}_{i}=0, i=1, \ldots, s$. Let us write $S:=\{1, \ldots, s\}, N:=\{s+1, \ldots, n\}$, and accordingly, for any $x \in \mathbb{R}^{n}, x_{S}:=\left(x_{1}, \ldots, x_{s}\right)^{\prime}$ and $x_{N}:=\left(x_{s+1}, \ldots, x_{n}\right)^{\prime}$. In the cases when $S=\emptyset$ and $N=\emptyset$, we understand $x_{N}=x$ and $x_{S}=x$, respectively. By using this notation, all coordinates of $\bar{x}_{S}$ are positive, while $\bar{z}_{S}=0_{s}$ and $\bar{x}_{N}=0_{n-s}$.

Theorem 4 Let $\left\{\left(x^{r}, y^{r}, z^{r}\right)\right\}_{r \in \mathbb{N}}$ be a sequence generated by the previous primal-dual path-following method associated with a sequence of nonnegative scalars $\left\{\mu_{r}\right\}_{r \in \mathbb{N}}$ converging to zero (where each $\mu_{r}$ may be defined in terms of $\left.\left\{\left(x^{j}, y^{j}, z^{j}\right)\right\}_{j=1}^{r}\right)$; i.e., such that $\left(x^{r+1}, y^{r+1}, z^{r+1}\right)$ is a solution of (37). Assume that $\left\{\left(x^{r}, y^{r}, z^{r}\right)\right\}_{r}$ converges to a certain $(\bar{x}, \bar{y}, \bar{z})$. Then, setting

$$
\kappa:=\operatorname{clm} \mathcal{N}\left(0_{n},(\bar{x}, \bar{y}, \bar{z})\right),
$$

for every $\varepsilon>0$ there exists $r_{0} \in \mathbb{N}$ such that for all $r \geq r_{0}$ we have

$$
d\left(\left(x^{r+1}, y^{r+1}, z^{r+1}\right), \Lambda\right) \leq(\kappa+\varepsilon) \delta_{r},
$$

where

$$
\begin{aligned}
\delta_{r}:= & \left\|z_{N}^{r}-\bar{z}_{N}\right\|\left\|x_{N}^{r+1}\right\|+\left\|z_{N}^{r}-z_{N}^{r+1}\right\|\left\|x_{N}^{r}\right\| \\
& +\left\|x_{S}^{r}-\bar{x}_{S}\right\|\left\|z_{S}^{r+1}\right\|+\left\|x_{S}^{r}-x_{S}^{r+1}\right\|\left\|z_{S}^{r}\right\|+2 \mu_{r} \sqrt{n},
\end{aligned}
$$

with all norms being the Euclidean ones in the respective spaces.

Proof For each $r$,

$$
\left(x^{r+1}, y^{r+1}, z^{r+1}\right) \in \mathcal{N}\left(\bar{Z} x^{r+1}+\bar{X} z^{r+1}\right) .
$$

Since $\kappa+\varepsilon$ is a calmness constant for $\mathcal{N}$ at $\left(0_{n},(\bar{x}, \bar{y}, \bar{z})\right)$ and $\bar{Z} x^{r+1}+\bar{X} z^{r+1} \rightarrow 0_{n}$, for $r$ large enough (say $r \geq r_{0}$ ) we have

$$
d\left(\left(x^{r+1}, y^{r+1}, z^{r+1}\right), \Lambda\right) \leq(\kappa+\varepsilon)\left\|\bar{Z} x^{r+1}+\bar{X} z^{r+1}\right\|_{\infty} \text { for all } r \geq r_{0} .
$$

Clearly we also have (recalling that $\|\cdot\|$ denotes the Euclidean norm)

$$
\left\|\bar{Z} x^{r+1}+\bar{X} z^{r+1}\right\|_{\infty} \leq\left\|\bar{Z}_{N} x_{N}^{r+1}\right\|+\left\|\bar{X}_{S} z_{S}^{r+1}\right\|, \text { for all } r \in \mathbb{N} .
$$


Since $X_{S}^{r} z_{S}^{r}=Z_{S}^{r} x_{S}^{r}$ and $X_{S}^{r} z_{S}^{r}=Z_{S}^{r} x_{S}^{r+1}+X_{S}^{r} z_{S}^{r+1}-\mu_{r} e_{S}$, see (38),

$$
\begin{aligned}
\left\|\bar{X}_{S} z_{S}^{r+1}\right\| & =\left\|\bar{X}_{S} z_{S}^{r+1}-X_{S}^{r} z_{S}^{r}+X_{S}^{r} z_{S}^{r}\right\| \\
& =\left\|\bar{X}_{S} z_{S}^{r+1}-Z_{S}^{r} x_{S}^{r+1}-X_{S}^{r} z_{S}^{r+1}+\mu_{r} e_{S}+X_{S}^{r} z_{S}^{r}\right\| \\
& =\left\|\left(\bar{X}_{S}-X_{S}^{r}\right) z_{S}^{r+1}-Z_{S}^{r} x_{S}^{r+1}+\mu_{r} e_{S}+Z_{S}^{r} x_{S}^{r}\right\| \\
& =\left\|\left(\bar{X}_{S}-X_{S}^{r}\right) z_{S}^{r+1}+Z_{S}^{r}\left(x_{S}^{r}-x_{S}^{r+1}\right)+\mu_{r} e_{S}\right\| \\
& \leq\left\|\bar{x}_{S}-x_{S}^{r}\right\|\left\|z_{S}^{r+1}\right\|+\left\|z_{S}^{r}\right\|\left\|x_{S}^{r}-x_{S}^{r+1}\right\|+\mu_{r} \sqrt{n} .
\end{aligned}
$$

By switching $x$ and $z$, and replacing subscript $S$ with $N$ we obtain

$$
\left\|\bar{Z}_{N} x_{N}^{r+1}\right\| \leq\left\|z_{N}^{r}-\bar{z}_{N}\right\|\left\|x_{N}^{r+1}\right\|+\left\|z_{N}^{r}-z_{N}^{r+1}\right\|\left\|x_{N}^{r}\right\|+\mu_{r} \sqrt{n} .
$$

The last result of the section quantifies the superlinear convergence of the method, under uniqueness of primal-dual solution, in terms of the calmness modulus of $\mathcal{N}$ for an appropriate choice of $\left\{\mu_{r}\right\}_{r \in \mathbb{N}}$. In order to integrate these results into the literature, the reader is addressed, for instance, to [31] and [36]. Paper [31] establishes the superlinear convergence of the iterates produced by a certain primal-dual interior-point method under some assumptions imposed to the sequence of differences $\left\{\left(x^{r+1}, z^{r+1}\right)-\left(x^{r}, z^{r}\right)\right\}$, while [36] deals with the speed of convergence of the sequence of the so-called duality gaps $\left\{\left(x^{r}\right)^{\prime} z^{r}\right\}$. Specifically, the superlinear convergence of such a sequence is established under some assumptions which include the strict complementarity of solution $(\bar{x}, \bar{y}, \bar{z})$; i.e., $\min _{1 \leq i \leq n}\left\{\bar{x}_{i}+\bar{z}_{i}\right\}>0$; adding the hypothesis that $\bar{x}$ is a non-degenerate vertex (i.e., the rank of $A$ is $m$ and $\bar{x}$ has $m$ positive coordinates), the authors derive the quadratic convergence of $\left\{\left(x^{r}\right)^{\prime} z^{r}\right\}$. Recall that it is well-known that strict complementarity together with non-degeneracy implies uniqueness of primal-dual solutions in linear programming, which connects with the following corollary. For completeness, let us also point out that the uniqueness of primal optimal solution also appears in different studies of the stability (e.g., continuity or Lipschitzian properties) of the linear optimization problem under perturbations of the data; see, e.g., [8, Theorem 16] and references therein.

Corollary 3 With the notation of the previous theorem, let

$$
\mu_{r}:=n^{-1 / 2}\left(\max _{1 \leq i \leq n}\left\{x_{i}^{r} z_{i}^{r}\right\}\right)^{2} \text { for all } r \in \mathbb{N} .
$$

If $\mu_{r}=0$ for some $r$, then the algorithm attains $\Lambda$ at step $r$. Otherwise, assume $\Lambda=\{(\bar{x}, \bar{y}, \bar{z})\}$, fix any $\varepsilon>0$ with $2(\kappa+\varepsilon) \varepsilon<1$, and let $r_{0} \in \mathbb{N}$ be such that $\max \left\{\left\|x_{N}^{r}\right\|,\left\|z_{S}^{r}\right\|\right\}<\varepsilon,\left\|x_{S}^{r}\right\| \leq\left\|\bar{x}_{S}\right\|+\varepsilon,\left\|z_{N}^{r}\right\| \leq\left\|\bar{z}_{N}\right\|+\varepsilon$ and (40) hold for all $r \geq r_{0}$. Then, for all $r \geq r_{0}$ we have

$$
\left\|\left(x^{r+1}, y^{r+1}, z^{r+1}\right)-(\bar{x}, \bar{y}, \bar{z})\right\| \leq \widetilde{\varepsilon}\left\|\left(x^{r}, y^{r}, z^{r}\right)-(\bar{x}, \bar{y}, \bar{z})\right\|,
$$

where

$$
\widetilde{\varepsilon}:=\frac{\left(4+2 \max \left\{\left(\left\|\bar{x}_{S}\right\|+\varepsilon\right)^{2},\left(\left\|\bar{z}_{N}\right\|+\varepsilon\right)^{2}\right\}\right)(\kappa+\varepsilon) \varepsilon}{1-2(\kappa+\varepsilon) \varepsilon} .
$$


Proof If $\mu_{r}=0$, for some $r$, then $\left(x^{r}, y^{r}, z^{r}\right)$ is a solution of (36), and then $\left(x^{r}, y^{r}, z^{r}\right) \in$ $\Lambda$; see Remark 8. Otherwise, under our current assumptions, recalling that $\bar{z}_{S}=0_{s}, \bar{x}_{N}=$ $0_{n-s}$, and that all norms below are the Euclidean (although the number of coordinates is not always the same), we have from (40) and our choice of $\mu_{r}$ that

$$
\begin{aligned}
& \left\|\left(x^{r+1}, y^{r+1}, z^{r+1}\right)-(\bar{x}, \bar{y}, \bar{z})\right\| \\
\leq & (\kappa+\varepsilon) \varepsilon\left(2\left\|z_{N}^{r}-\bar{z}_{N}\right\|+\left\|\bar{z}_{N}-z_{N}^{r+1}\right\|\right)+(\kappa+\varepsilon) \varepsilon\left(2\left\|x_{S}^{r}-\bar{x}_{S}\right\|+\left\|\bar{x}_{S}-x_{S}^{r+1}\right\|\right) \\
& +2(\kappa+\varepsilon) \max \left\{\left\|z_{S}^{r}-\bar{z}_{S}\right\|^{2}\left(\max _{1 \leq i \leq s} x_{i}^{r}\right)^{2},\left\|x_{N}^{r}-\bar{x}_{N}\right\|^{2}\left(\max _{s+1 \leq i \leq n} z_{i}^{r}\right)^{2}\right\} \\
\leq & (\kappa+\varepsilon) \varepsilon\left(2\left\|z^{r}-\bar{z}\right\|+2\left\|x^{r}-\bar{x}\right\|+\left\|\bar{z}-z^{r+1}\right\|+\left\|\bar{x}-x^{r+1}\right\|\right) \\
& +2(\kappa+\varepsilon) \max \left\{\varepsilon\left\|z_{S}^{r}-\bar{z}_{S}\right\|\left\|x_{S}^{r}\right\|^{2}, \varepsilon\left\|x_{N}^{r}-\bar{x}_{N}\right\|\left\|z_{N}^{r}\right\|^{2}\right\} \\
\leq & 4(\kappa+\varepsilon) \varepsilon\left\|\left(x^{r}, y^{r}, z^{r}\right)-(\bar{x}, \bar{y}, \bar{z})\right\|+2(\kappa+\varepsilon) \varepsilon\left\|\left(x^{r+1}, y^{r+1}, z^{r+1}\right)-(\bar{x}, \bar{y}, \bar{z})\right\| \\
& +2(\kappa+\varepsilon) \varepsilon \max \left\{\left(\left\|\bar{x}_{S}\right\|+\varepsilon\right)^{2},\left(\left\|\bar{z}_{N}\right\|+\varepsilon\right)^{2}\right\}\left\|\left(x^{r}, y^{r}, z^{r}\right)-(\bar{x}, \bar{y}, \bar{z})\right\|,
\end{aligned}
$$

for all $r \geq r_{0}$. Then (41) follows immediately.

We finish this section with an illustrative example focussed on the computation of $\kappa$. Observe that the constraint system in (39) fits the model (34) with $I=\emptyset$; although, for simplicity, in this example we do not split into two inequalities each of the unperturbed equalities in the second and third row blocks of (39), namely, $A x=b$ and $A^{\prime} y+z=c$; see Remark 4.

Example 6 Let us consider the primal-dual problem (35) with

$$
A=\left(\begin{array}{ll}
2 & 1
\end{array}\right), b=4, c=\left(\begin{array}{l}
2 \\
2
\end{array}\right) .
$$

The reader can easily check that there is a unique primal-dual solution $(\bar{x}, \bar{y}, \bar{z})$, with $\bar{x}=\left(\begin{array}{l}2 \\ 0\end{array}\right), \bar{y}=1, \bar{z}=\left(\begin{array}{l}0 \\ 1\end{array}\right)$. The constraint system defining $\mathcal{N}\left(0_{2}\right)$ in (39) is

$$
\left(\begin{array}{cc|c|cc}
0 & 0 & 0 & 2 & 0 \\
0 & 1 & 0 & 0 & 0 \\
\hline 2 & 1 & 0 & 0 & 0 \\
\hline 0 & 0 & 2 & 1 & 0 \\
0 & 0 & 1 & 0 & 1 \\
\hline-1 & 0 & 0 & 0 & 0 \\
0 & -1 & 0 & 0 & 0 \\
\hline 0 & 0 & 0 & -1 & 0 \\
0 & 0 & 0 & 0 & -1
\end{array}\right)\left(\begin{array}{c}
x_{1} \\
x_{2} \\
y \\
z_{1} \\
z_{2}
\end{array}\right) \begin{cases}=0, t=1 \in E, \\
=0, & t=2 \in E, \\
=4, & t=3 \in U, \\
=2, & t=4 \in U, \\
=2, & t=5 \in U, \\
\leq 0, & t=6 \in U, \\
\leq 0, & t=7 \in U, \\
\leq 0, & t=8 \in U, \\
\leq 0, & t=9 \in U .\end{cases}
$$

Here we have left the unperturbed equalities associated with $t \in\{3,4,5\}$ as equalities according to Remark 4. Then $I=\emptyset, E=\{1,2\}, U(\bar{x}, \bar{y}, \bar{z})=\{3,4,5,7,8\}$. The set $\mathcal{D}(\bar{x}, \bar{y}, \bar{z})$ consists of nine elements, but only four of them are maximal with respect to the (coordinatewise) inclusion order -see Remark 2-, namely the maximal $\left(D_{1}, D_{2}^{+}, D_{2}^{-}, D_{3}\right)$ 
are:

$$
\begin{array}{ll}
(\emptyset, \emptyset, \emptyset,\{3,4,5,7,8\}), & (\emptyset,\{1\}, \emptyset,\{3,4,5,7\}), \\
(\emptyset,\{2\}, \emptyset,\{3,4,5,8\}), & (\emptyset,\{1,2\}, \emptyset,\{3,4,5\}) .
\end{array}
$$

Moreover, $\min _{\left(D_{1}, D_{2}^{+}, D_{2}^{-}, D_{3}\right) \in \mathcal{D}(\bar{x})} \delta\left(D_{1}, D_{2}^{+}, D_{2}^{-}, D_{3}\right)$ in Corollary 2 is attained at $(\emptyset,\{1,2\}, \emptyset,\{3,4,5\})$ and equals, looking at the rows of the coefficient matrix in (42) and recalling that $\mathbb{R}^{5}$ is considered to be endowed with the Euclidean norm,

$$
d_{*}\left(0_{n}, \operatorname{conv}\left\{a_{1}, a_{2}\right\}+\operatorname{span}\left\{a_{3}, a_{4}, a_{5}\right\}\right)=\frac{2 \sqrt{2}}{\sqrt{13}},
$$

so that $\kappa=\sqrt{13} /(2 \sqrt{2})$.

Acknowledgements The authors wish to thank the anonymous referees for their valuable critical comments which have definitely improved the first version of the manuscript.

Funding This research has been partially supported by Grant PGC2018-097960-B-C21 from MICINN, Spain, and ERDF, "A way to make Europe", European Union.

Open Access This article is licensed under a Creative Commons Attribution 4.0 International License, which permits use, sharing, adaptation, distribution and reproduction in any medium or format, as long as you give appropriate credit to the original author(s) and the source, provide a link to the Creative Commons licence, and indicate if changes were made. The images or other third party material in this article are included in the article's Creative Commons licence, unless indicated otherwise in a credit line to the material. If material is not included in the article's Creative Commons licence and your intended use is not permitted by statutory regulation or exceeds the permitted use, you will need to obtain permission directly from the copyright holder. To view a copy of this licence, visit http://creativecommons.org/licenses/by/4.0/.

\section{References}

1. Al-Jeiroudi, G., Gondzio, J., Hall, J.: Preconditioning indefinite systems in interior point methods for large scale linear optimization. Optim. Methods Softw. 23, 345-363 (2008)

2. Bai, K., Ye, J.J., Zhang, J.: Directional quasi/pseudo-normality as sufficient conditions for metric subregularity. SIAM J. Optim. 29, 2625-2649 (2019)

3. Bazaraa, M.S., Sherali, H.D., Shetty, C.M.: Nonlinear Programming: Theory and Algorithms. Wiley, New York (1993)

4. Bertsimas, D., Tsitsiklis, J.N.: Introduction to Linear Optimization, Athena Scientific, Dynamic Ideas, Belmont, Massachusetts (1997)

5. Cánovas, M.J., Gómez-Senent, F.J., Parra, J.: Regularity modulus of intersection mappings. Application to the stability of squations via splitting into inequalities. J. Convex Anal. 19, 913-926 (2012)

6. Cánovas, M.J., Hall, J.A.J., López, M.A., Parra, J.: Calmness of partially perturbed linear systems with an application to the central path. Optimization 68, 465-483 (2019)

7. Cánovas, M.J., Henrion, R., López, M.A., Parra, J.: Outer limits of subdifferentials and calmness moduli in linear and nonlinear programming. J. Optim. Theory Appl. 169, 925-952 (2016)

8. Cánovas, M.J., Klatte, D., López, M.A., Parra, J.: Metric regularity in convex semi-infinite optimization under canonical perturbations. SIAM J. Optim. 18, 717-732 (2007)

9. Cánovas, M.J., López, M.A., Mordukhovich, B.S., Parra, J.: Quantitative stability of linear inequality systems under block perturbations with applications to convex systems. Top 20, 310-327 (2012)

10. Cánovas, M.J., López, M.A., Mordukhovich, B.S., Parra, J.: Subdifferentials and stability analysis of feasible set and Pareto front mappings in linear multiobjective optimization. Vietnam J. Math 48, 315334 (2020)

11. Cánovas, M.J., López, M.A., Parra, J., Toledo, F.J.: Calmness of the feasible set mapping for linear inequality systems. Set-Valued Var Anal. 22, 375-389 (2014) 
12. Cánovas, M.J., Parra, J., Rückmann, J.-J., Toledo, F.J.: Point-based neighborhoods for sharp calmness constants in linear programming. Set-Valued Var. Anal. 25, 757-772 (2017)

13. Dontchev, A.L., Rockafellar, R.T.: Implicit Functions Mappings, Solution: A View from Variational Analysis. Springer, New York (2009)

14. Facchinei, F., Fischer, A., Herrich, M.: An LP-Newton method: nonsmooth equations, KKT systems, and nonisolated solutions. Math. Program, Ser. A 146, 1-36 (2014)

15. Goberna, M.A., López, M.A.: Linear Semi-Infinite Optimization. John Wiley \& Sons, Chichester (UK) (1998)

16. Gondzio, J.: Interior point methods 25 years later. Eur. J. Oper. Res. 218, 587-601 (2012)

17. Hu, H.: Characterizations of the strong basic constraint qualifications. Math Oper Res. 30, 956-965 (2005)

18. Hoffman, A.J.: On approximate solutions of systems of linear inequalities. J. Res. Natl. Bur. Stand. 49, 263-265 (1952)

19. Ioffe, A.D.: Variational Analysis of Regular Mappings. Springer, Cham (2017)

20. Karmarkar, N.: A new polynomial-time algorithm for linear programming. Combinatorica 4(4), 373-395 (1984)

21. Klatte, D., Kummer, B.: Nonsmooth Equations in Optimization: Regularity, Calculus, Methods and Applications, Nonconvex Optim Appl, vol. 60. Kluwer Academic, Dordrecht, The Netherlands (2002)

22. Klatte, D., Kummer, B.: Optimization methods stability of inclusions in Banach spaces. Math Program. B 117, 305-330 (2009)

23. Klatte, D., Kummer, B.: Approximations and generalized Newton methods. Math. Program. Ser. B 168 673-716 (2018)

24. Kruger, A., Ngai, H.V.A.N., Théra, M.: Stability of error bounds for convex constraint systems in Banach spaces. SIAM J. Optim. 20, 3280-3296 (2010)

25. Li, M.H., Meng, K.W., Yang, X.Q.: On error bound moduli for locally Lipschitz and regular functions. Math. Program. Ser. A 171, 463-487 (2018)

26. Mangasarian, O.L., Fromovitz, S.: The Fritz John Necessary Optimality Conditions in the Presence of Equality and Inequality Constraints. J. Math. Anal. Appl. 17, 37-47 (1967)

27. Megiddo, N. In: Megiddo, N. (ed.): Pathways to the Optimal Set in Linear Programming, in Progress in Mathematical Programming, pp. 131-158. Springer-Verlag, New York (1989)

28. Monteiro, R.D.C., Adler, I.: Interior path following primal-dual algorithms. Part I: Linear programming. Math. Program 44, 27-41 (1989)

29. Mordukhovich, B.S.: Variational Analysis and Generalized Differentiation, I: Basic Theory. Springer, Berlin (2006)

30. Potra, F.A., Wright, S.J., Interior-point methods: J. Comput. Appl. Math. 124, 281-302 (2000)

31. Potra, F.A.: Q-superlinear convergence of the iterates in primal-dual interior-point methods. Math. Program., Ser. A 91, 99-115 (2001)

32. Renegar, J.: A polynomial time algorithm based on Newton's method for linear programming. Math. Program 40, 59-93 (1988)

33. Rockafellar, R.T.: Convex Analysis. Princeton University Press, Princeton, N.J. (1970)

34. Rockafellar, R.T., Wets, R.J.-B.: Variational Analysis. Springer, Berlin (1998)

35. Wang, X., Ye, J.J., Yuan, X., Zeng, S., Zhang, J.: Perturbation techniques for convergence analysis of proximal gradient method and other first-order algorithms via variational analysis. Set-Valued Var. Anal. https://doi.org/10.1007/s11228-020-00570-0 (2021)

36. Zhang, Y., Tapia, R.A., Dennis, J.E.: On the superlinear and quadratic convergence of primal-dual interior-point linear programming algorithms. SIAM J. Optim. 2, 304-324 (1992)

Publisher's Note Springer Nature remains neutral with regard to jurisdictional claims in published maps and institutional affiliations. 